\title{
Collider and Dark Matter Phenomenology of Models with Mirage Unification
}

\author{
Howard Baer ${ }^{a, b}$, Eun-Kyung Park ${ }^{a}$, Xerxes Tata ${ }^{c}$ and Ting T. Wang ${ }^{a, b}$ \\ ${ }^{a}$ Department of Physics, Florida State University, Tallahassee, FL 32306, USA \\ ${ }^{b}$ Department of Physics, University of Wisconsin, Madison, WI, 53706, USA \\ ${ }^{c}$ Department of Physics and Astronomy, University of Hawaii, Honolulu, HI 96822, USA \\ E-mail: baer@hep.fsu.edu, epark@hep.fsu.edu, \\ tata@phys.hawaii.edu, tingwang@hep.fsu.edu
}

\begin{abstract}
We examine supersymmetric models with mixed modulus-anomaly mediated SUSY breaking (MM-AMSB) soft terms which get comparable contributions to SUSY breaking from moduli-mediation and anomaly-mediation. The apparent (mirage) unifica-

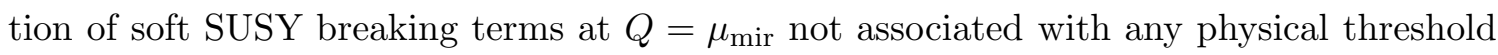
is the hallmark of this scenario. The MM-AMSB structure of soft terms arises in models of string compactification with fluxes, where the addition of an anti-brane leads to an uplifting potential and a de Sitter universe, as first constructed by Kachru et al.. The phenomenology mainly depends on the relative strength of moduli- and anomaly-mediated SUSY breaking contributions, and on the Higgs and matter field modular weights, which are determined by the location of these fields in the extra dimensions. We delineate the allowed parameter space for a low and high value of $\tan \beta$, for a wide range of modular weight choices. We calculate the neutralino relic density and display the WMAP-allowed regions. We show the reach of the CERN LHC and of the International Linear Collider. We discuss aspects of MM-AMSB models for Tevatron, LHC and ILC searches, muon $g-2$ and $b \rightarrow s \gamma$ branching fraction. We also calculate direct and indirect dark matter detection rates, and show that almost all WMAP-allowed models should be accessible to a ton-scale noble gas detector. Finally, we comment on the potential of colliders to measure the mirage unification scale and modular weights in the difficult case where $\mu_{\mathrm{mir}} \gg M_{\mathrm{GUT}}$.
\end{abstract}

KeYWords: Supersymmetry Phenomenology, Supersymmetric Standard Model, Dark Matter. 


\section{Introduction}

Superstring theory allows for a consistent merging of gravitational physics with quantum mechanics, while also containing within it the possibility of describing the gauge interactions of the Standard Model. This impressive theoretical framework does not, however, allow the extraction of predictions of physical phenomena at experimentally accessible energy scales. A major obstruction to obtaining a predictive superstring theory is the lack of understanding of how the degeneracy associated with the many flat directions in the space of scalar fields (the moduli) is lifted to yield the true ground state, the problem being that many couplings necessary for the extraction of "observables quantities" are determined by the ground state values of these moduli.

The discovery of a new class of compactifications, where the extra spatial dimensions are curled up to small sizes with fluxes of additional fields trapped along these extra dimensions has been exploited by Kachru et al. (KKLT) 11 to construct a concrete model with a stable, calculable ground state with a positive cosmological constant and broken supersymmetry. This toy model is based on type-IIB superstrings including compactification of the extra dimensions to a Calabi-Yau orientifold, with fluxes along these extra directions. While the background fluxes serve to stabilize the dilaton and the moduli that determine the shape of the compact manifold, it is necessary to invoke a non-perturbative mechanism such as gaugino condensation on a $D 7$ brane to stabilize the size of the compact manifold. Finally, a non-supersymmetric anti-brane $(\overline{D 3})$ - included in order to break supersymmmetry - also yields a de Sitter universe as required by observations. The KKLT construction, which yields an example of a low energy theory that has no unwanted light moduli, broken supersymmetry and a positive cosmological constant, may be viewed as a starting point for the program of discovering a string ground state that may lead to the (supersymmetric) Standard Model at low energies, and which is consistent with various constraints from cosmology.

These considerations have recently motivated several authors to analyze the structure of the soft SUSY breaking (SSB) terms in models based on a generalization of the KKLT set-up[2]. The key observation is that because of the mass hierarchy

$$
m_{\text {moduli }} \gg m_{3 / 2} \gg m_{\mathrm{SUSY}}
$$

that develops in these models, the SSB terms receive comparable contributions via both modulus (gravity) and anomaly mediation of SUSY breaking[3], with their relative size parametrized by one new parameter $\alpha$. The hierarchy (1.1) that leads to mixed modulusanomaly mediated SUSY breaking seemingly allows the moduli to decay early enough not to disrupt Big Bang nucleosynthesis. It has, however, been pointed out that these decays of the moduli would inevitably produce an unacceptably large number of gravitinos (or other sparticles) [] which would subsequently decay to the LSP, unless the gravitino is itself heavier than $\sim 100 \mathrm{TeV}$, or the density of particles is reduced by some mechanism such as a period of thermal inflation [5]. Here, we will assume that such a mechanism is operative and that the observed dark matter, in our case the lightest neutralino, is produced thermally upon subsequent reheating. Upon integrating out the heavy dilaton field and the 
shape moduli, we are left with an effective broken supergravity theory of the observable sector fields denoted by $\hat{Q}$ and the size modulus field $\hat{T}$. The Kähler potential depends on the location of matter and Higgs superfields in the extra dimensions via their modular weights $n_{i}=0$ (1) for matter fields located on $D 7$ (D3) branes, or $n_{i}=1 / 2$ for chiral multiplets on brane intersections, while the gauge kinetic function $f_{a}=\hat{T}^{l_{a}}$, where $a$ labels the gauge group, is determined by the corresponding location of the gauge supermultiplets, since the power $l_{a}=1$ (0) for gauge fields on $D 7(D 3)$ branes [6]. ${ }^{1}$

Within the MM-AMSB model, the SSB gaugino mass parameters, trilinear SSB parameters and sfermion mass parameters, all renormalized just below the unification scale (taken to be $Q=M_{\mathrm{GUT}}$ ), are given (in the convention used in the event generator Isajet [10]) by,

$$
\begin{aligned}
M_{a} & =M_{s}\left(l_{a} \alpha+b_{a} g_{a}^{2}\right), \\
A_{i j k} & =M_{s}\left(-a_{i j k} \alpha+\gamma_{i}+\gamma_{j}+\gamma_{k}\right), \\
m_{i}^{2} & =M_{s}^{2}\left(c_{i} \alpha^{2}+4 \alpha \xi_{i}-\dot{\gamma}_{i}\right),
\end{aligned}
$$

where $M_{s} \equiv \frac{m_{3 / 2}}{16 \pi^{2}}, b_{a}$ are the gauge $\beta$ function coefficients for gauge group $a$ and $g_{a}$ are the corresponding gauge couplings. The coefficients that appear in (1.2)-(1.4) are given by $c_{i}=1-n_{i}, a_{i j k}=3-n_{i}-n_{j}-n_{k}$ and $\xi_{i}=\sum_{j, k} a_{i j k} \frac{y_{i j k}^{2}}{4}-\sum_{a} l_{a} g_{a}^{2} C_{2}^{a}\left(f_{i}\right)$. Finally, $y_{i j k}$ are the superpotential Yukawa couplings, $C_{2}^{a}$ is the quadratic Casimir for the $\mathrm{a}^{\text {th }}$ gauge group corresponding to the representation to which the sfermion $\tilde{f}_{i}$ belongs, $\gamma_{i}$ is the anomalous dimension and $\dot{\gamma}_{i}=8 \pi^{2} \frac{\partial \gamma_{i}}{\partial \log Q}$. Expressions for the last two quantities involving the anomalous dimensions can be found in the Appendix of Ref. [11], whose notation we adopt here. ${ }^{2}$

The MM-AMSB model is completely specified by the parameter set,

$$
m_{3 / 2}, \alpha, \tan \beta, \operatorname{sign}(\mu), n_{i}, l_{a} .
$$

The mass scale for the MSSM SSB parameters is dictated by $M_{s} \equiv \frac{m_{3 / 2}}{16 \pi^{2}}$. The phenomenological parameter $\alpha$, which could be of either sign, determines the relative contributions of anomaly mediation and gravity mediation to the soft terms, and as mentioned above $|\alpha| \sim \mathcal{O}(1)$ is the hallmark of this scenario. Non-observation of large flavor changing neutral currents implies common modular weights of particles with the same gauge quantum numbers: within this framework, this suggests a common location for these fields in the extra dimensions. Grand Unification implies matter particles within the same GUT multiplet have common modular weights, and that the $l_{a}$ are universal. We will assume that all $l_{a}=l$ and, for simplicity, a common modular weight for all matter particles, but allow a different (common) one for the two Higgs doublets of the MSSM.

\footnotetext{
${ }^{1}$ More specifically, these modular weights for chiral superfields are obtained for examples with toroidal compactifications, possibly with singularities [7]. This is not generic as Calabi-Yau compactifications allow for more general choices: for instance $n_{i}=\frac{2}{3}$ may also be allowed (see Refs. 8, 9]).

${ }^{2}$ We note that $\alpha$ defined in Ref. [6] differs from the definition in Ref. [11] that we use here by $\alpha_{\text {Ref. } 6 \text { [ }}=$ $\frac{16 \pi^{2}}{\ln \left(M_{P} / m_{\frac{3}{2}}\right)} \frac{1}{\alpha_{\text {our }}}$. The original KKLT construction yields $\alpha_{\text {Ref. }} \mid 6$ | $\simeq 1$, corresponding to $\alpha \sim 4.6$ for $m_{3 / 2} \sim$ $1 \mathrm{TeV}$, in the notation used in this paper.
} 
The universality of the $l_{a}$ leads to the phenomenon of mirage unification [6, 11] of gaugino masses. In mirage unification, the splitting of the gaugino masses at $Q=M_{\mathrm{GUT}}$ is proportional to $\beta_{a}\left(g_{a}\right) / g_{a}$, where $\beta_{a}$ are the beta-functions of their associated gauge groups. As the gaugino masses run from $M_{\mathrm{GUT}}$ to lower energy scales, the RG running exactly cancels the GUT scale mass splitting, leading (for $\alpha>0$ ) to unified gaugino masses at some intermediate energy scale $Q=\mu_{\text {mir }} \neq M_{\mathrm{GUT}}$, the scale of unification of gauge couplings. Indeed, the observation of gaugino unification at the mirage unification scale,

$$
\mu_{\mathrm{mir}}=M_{\mathrm{GUT}} e^{-8 \pi^{2} /(l \alpha)}
$$

is the smoking gun of the MM-AMSB scenario. If $\alpha<0, \mu_{\text {mir }}>M_{\mathrm{GUT}}$, though one would have to continue extrapolation still using MSSM RGEs beyond $M_{\mathrm{GUT}}$ to discover this! We will assume hereafter that $l \neq 0$, since $l=0$ would be distinguished by a gaugino mass pattern as in the AMSB framework. While $\mu_{\text {mir }}$ determines $l \alpha$, the (unified) value of the the gaugino masses extrapolated to $Q=\mu_{\text {mir }}$ is $M_{a}\left(\mu_{\text {mir }}\right)=M_{s} \times(l \alpha)$, and so gives the value of $M_{s}$ (and hence $m_{3 / 2}$ ). We note here that the soft SUSY breaking scalar masses of the first two generations also unify at $Q=\mu_{\text {mir }}$, which allows for experimental corroboration of mirage unification of gaugino masses. In fact, for cases with $n_{H}+2 n_{m}=2$, third generation and Higgs SSB terms also unify at $\mu_{\text {mir }}[6]$.

Phenomenologically attractive features of the MM-AMSB scenario, also referred to as the mirage mediation model, are that it provides natural solutions to 1) the negative slepton mass squared problem inherent to AMSB models, 2) the SUSY flavor problem, plausibly assuming common location for matter fermions in the extra dimensions, and the concomitant universality of their modular weights, and 3) the SUSY CP problem, in that (up to $\mathcal{O}\left(\frac{1}{4 \pi^{2}}\right)$ corrections) there are no physical $C P$ violating phases in $\mu$, gaugino masses and $A$-terms: moreover, there are mechanisms that also yield a real value for $b \equiv B \mu[6,9]$.

For these reasons, a number of authors have begun exploring the associated collider and dark matter phenomenology of these models. Indeed, in Ref. [6] the phenomena of mirage unification was noted, while mass spectra were computed in Ref's [6, 11, 12, [13]. In Ref. [14], it was emphasized that bringing the mirage unification scale down close to the weak scale ameliorates fine-tuning problems in supersymmetric theories. In Ref. [13], mass spectra were computed in the MM-AMSB scenario for two choices of modular weights $\left(n_{H}, n_{m}\right)=(0,0)$ and $\left(1, \frac{1}{2}\right)$. Regions of parameter space where the neutralino is the lightest supersymmetric particle, and where its predicted relic abundance (assuming it is a thermal relic in standard Big Bang cosmology) is in agreement with WMAP measurements 15 were mapped out. Allowing that the observed DM may consist of more than one component, $\Omega_{\widetilde{Z}_{1}} h^{2} \lesssim 0.13$. Collider reaches in MM-AMSB parameter space were also presented. In Ref. [16], aspects of LHC detection for MM-AMSB spectra where sparticle masses are roughly degenerate were examined. In Ref. [17, it was pointed out that measurement of soft SUSY breaking terms at the LHC and ILC could measure the matter field and in some cases the Higgs field modular weights, by taking ratios of scalar to gaugino masses at $\mu_{\mathrm{mir}}$. In Ref. [18], the neutralino relic abundance and direct and indirect dark matter detection rates were presented for four modular weight choices. 
In this paper, we present allowed parameter space regions for a wide range of Higgs and matter field modular weights at low and high $\tan \beta$ values. We also compute the neutralino relic abundance, and note where it is consistent with WMAP measurements. We present the approximate reach of the CERN LHC with $100 \mathrm{fb}^{-1}$ of integrated luminosity, and the reach of a $\sqrt{s}=0.5$ and $1 \mathrm{TeV}$ international linear $e^{+} e^{-}$collider (ILC). We organize the parameter space discussion and delineate the WMAP allowed regions in Sec. 2. In Secs. 3-6, we present various features of MM-AMSB models from a scan over a wide range of modular weight choices, confining ourselves to the regions consistent with WMAP measurements. In Sec. 3, we present plots of $(g-2)_{\mu}$ and the branching fraction for $b \rightarrow s \gamma$ decays. The value of $(g-2)_{\mu}$ is found to rule out most of the MM-AMSB parameter space at large negative values of $\alpha$ and $\mu>0$. In Sec. 4 , we comment on features of WMAP allowed MM-AMSB models relevant for SUSY searches at the Fermilab Tevatron, CERN LHC and the ILC. In Sec. 5, we discuss projections for signals via various direct and indirect searches for dark matter. We find that almost all WMAP-allowed parameter space should be accessible to ton-size direct dark matter search experiments. Signals from indirect searches (especially those from high energy gamma rays) are (very) sensitive to the dark matter halo profile in our Galaxy. An optimistic choice for this implies that GLAST would be sensitive to essentially the entire parameter space, but a different (yet currently viable) choice suggests that many models may be below the level of sensitivity for a wide range of $m_{\widetilde{Z}_{1}}$. In Sec. 6 , we extend our earlier discussion [17], and consider the possibility of determining mirage unification and matter modular weights in the case where $\alpha<0$ where $\mu_{\text {mir }}>M_{\mathrm{GUT}}$. In Sec. 7, we present our conclusions.

\section{WMAP-Allowed Parameter Space of MM-AMSB models}

To facilitate calculations within the MM-AMSB framework, we have incorporated it as model line number 9 in the Isajet 7.75 event generator 10]. For a given set of parameters (1.5), Isajet runs the measured gauge and Yukawa couplings from the weak scale to the GUT scale, where the GUT scale is determined by where gauge couplings $g_{1}=g_{2}$. The boundary conditions (1.2)-(1.4) are imposed at $Q=M_{\mathrm{GUT}}$, and the values of weak scale SSB parameters are obtained by numerically solving the complete set of 26 coupled 2-loop renormalization group equations. Sparticle mixing matrices are determined by freezing the parameters at a scale $Q=\sqrt{m_{\tilde{t}_{L}} m_{\tilde{t}_{R}}}$, while non-mixing soft parameters (that determine masses) are frozen out at a scale equal to their value[19]. Next, the RG-improved one-loop effective potential is minimized at an optimized scale (which accounts for leading two-loop terms), allowing the magnitude of $\mu$ to be determined, and complete one-loop radiative corrections to sparticle and Higgs masses are obtained. The Yukawa couplings are updated due to threshold effects. The SSB parameters evolved back to $Q=M_{\mathrm{GUT}}$, of course, do not match their input boundary values because of the differences in the upward and downward evolution. The procedure is iterated until a stable solution is obtained. The neutralino relic density $\Omega_{\widetilde{Z}_{1}} h^{2}, B F(b \rightarrow s \gamma),(g-2)_{\mu}, B F\left(B_{s} \rightarrow \mu^{+} \mu^{-}\right)$and the direct

dark matter detection cross section $\sigma\left(\widetilde{Z}_{1} p\right)$ are then obtained using the Isatools package [20]. We interface to DarkSUSY [21] to obtain indirect dark matter detection rates. 
Throughout our analysis, we take all the $l_{a}=1$, and assume that all matter fields have a common modular weight $n_{m}$, but allow the Higgs fields to have a different modular weight $n_{H}$ not necessarily equal to $n_{m}$. We organize our discussion of the models by specifying the values of $\left(n_{H}, n_{m}\right)$ where each of these is allowed to take the values, $0,1 / 2$ and 1 . The phenomenology of models with intermediate values $(1 / 3$ and $2 / 3)$ of modular weights that may be allowed by more general compactifications is presumably bracketed by the nine cases that we study. For any choice of $\tan \beta$ and the modular weights, the $\alpha-m_{3 / 2}$ plane provides a convenient panorama for an overview of the phenomenology. We begin by delineating the regions of this plane that are allowed by theoretical considerations and by the constraint on $\Omega_{\widetilde{Z}_{1}} h^{2}$ from WMAP, starting with the examination of the three $n_{m}$ cases with $n_{H}=0$.

\section{$2.1 \mathrm{n}_{\mathrm{H}}=0$ cases}

Our first results are presented in Fig. 1. Here we show the allowed parameter space in the $\alpha$ vs. $m_{3 / 2}$ plane for $n_{H}=0$ and $\left.\left.a\right) n_{m}=0, \tan \beta=10, b\right) n_{m}=0, \tan \beta=30$, c) $\left.\left.n_{m}=1 / 2, \tan \beta=10, d\right) n_{m}=1 / 2, \tan \beta=30 e\right), n_{m}=1, \tan \beta=10$ and $f$ ) $n_{m}=1, \tan \beta=30$. We take $\mu>0$ and $m_{t}=175 \mathrm{GeV}$ throughout, and require points to be compatible with approximate sparticle and Higgs mass constraints from LEP2 searches: $m_{\widetilde{W}_{1}}>103 \mathrm{GeV}, m_{\tilde{\tau}_{1}}>95 \mathrm{GeV}, m_{\widetilde{Z}_{1}}+m_{\widetilde{Z}_{2}}>120 \mathrm{GeV}$ and $m_{h}>110 \mathrm{GeV}$ [22]. ${ }^{3}$ The white unshaded regions do not lead to an acceptable sparticle mass spectrum due to a wrong pattern of electroweak symmetry breaking, signalled by tachyonic sfermion masses, a negative value of $\mu^{2}$ or one of the Higgs squared masses. The turquoise-shaded region leads to a top-squark LSP, while the magenta-shaded region leads to a stau LSP: these regions would likely give rise to stable colored or charged relics from the Big Bang, and hence are also excluded. The blue-dotted region leads to an acceptable sparticle mass spectrum with a neutralino LSP, but in this case the calculated relic density $\Omega_{\widetilde{Z}_{1}} h^{2}>0.5$, in violation of WMAP limits. The green-shaded region has a lower relic density, $0.13<\Omega_{\widetilde{Z}_{1}} h^{2}<0.5$ but is also excluded. The red-shaded regions have $\Omega_{\widetilde{Z}_{1}} h^{2}<0.13$ in accord with WMAP and hence are allowed. Frames $a$ ) and $b$ ) are repeated, but updated, from Ref. [13]. As noted in Ref. [13] (and also in Ref. [18] and the updated Ref. [11]), in frame $a$ ) the allowed red-shaded region for $\alpha>0$ occurs because the $\tilde{t}_{1}$ is quite light, with $m_{\tilde{t}_{1}} \sim m_{\widetilde{Z}_{1}}$. This is due to the large value of the $A_{t}$ parameter occurring in Eq. 1.3. A large $A_{t}$ feeds into the running of the soft SUSY breaking terms $m_{\tilde{t}_{L}}^{2}$ and $m_{\tilde{t}_{R}}^{2}$ via large values of $X_{t}=m_{\tilde{t}_{L}}^{2}+m_{\tilde{t}_{R}}^{2}+m_{H_{u}}^{2}+A_{t}^{2}$ in the corresponding RGEs, which then accentuates the impact of the large top-quark Yukawa coupling in driving these to small values. The resultant light top squark $\tilde{t}_{1}$ enhances neutralino annihilation in the early universe via top-squark co-annihilation. There is also a red-shaded region around $\alpha \sim-2$ where the weak scale gaugino masses $M_{1} \sim-M_{2}$, so that bino-wino co-annihilation (BWCA) acts to reduce the neutralino relic density 24] to the required level.

\footnotetext{
${ }^{3}$ While the LEP2 SM Higgs mass constraint $m_{h}>114.4 \mathrm{GeV}$ translates to a corresponding constraint on $h$ as long as $m_{A}$ is large, we require a somewhat lower bound owing to an expected 3-4 GeV uncertainty on the theory calculation of $m_{h}$; for a discussion of how this is affected if $m_{A} \lesssim 150-200 \mathrm{GeV}$, see Ref. 223.
} 
In frame $b$ ), for $\tan \beta=30$, once again we see a top-squark-coannihilation region adjacent to the turquoise-shaded stop-LSP region. But in this case, at large $\alpha$ and low $m_{3 / 2} \sim 5 \mathrm{TeV}$, there is also a region of stau-coannihilation. Note in frame $b$ ) that the WMAP-allowed BWCA region at $\alpha \sim-2$ has been eliminated. On these plots we also denote the approximate reach of the CERN LHC with $100 \mathrm{fb}^{-1}$ of integrated luminosity. The LHC reach has been evaluated in Ref. 25] and found to extend to $m_{\tilde{g}} \sim 3.1 \mathrm{TeV}$ in the case where $m_{\tilde{q}} \simeq m_{\tilde{g}}$ (as is the case for MM-AMSB models, see Sec. 4). ${ }^{4}$ The 100 $\mathrm{fb}^{-1}$ LHC reach extends up to $m_{3 / 2} \sim 60 \mathrm{TeV}$, essentially covering all the region with $m_{\tilde{g}} \sim m_{\tilde{q}} \lesssim 3.1 \mathrm{TeV}$. The reach of a $\sqrt{s}=0.5$ and $1 \mathrm{TeV}$ ILC is also indicated. The ILC reach is determined mainly by the kinematic limit for $\widetilde{W}_{1}^{+} \widetilde{W}_{1}^{-}$or $\tilde{\tau}_{1}^{+} \tilde{\tau}_{1}^{-}$pair production processes [26], and is somewhat smaller than the reach of the CERN LHC.

In frame $c$ ), we see how the allowed parameter space changes if instead we take matter to live on the $D 3-D 7$ brane intersection, for which the modular weight $n_{m}=1 / 2$. A value of $n_{m}=1 / 2$ acts to reduce the magnitude of the $A_{i}$ parameters in Eq. (1.3), and to reduce the moduli contribution to the matter scalar masses in Eq. (1.4). As a result of the reduced value of $A_{t}$ at $M_{\mathrm{GUT}}$, there is not so strong of an RG push on the top squark soft masses, and so we are left with somewhat heavier top squarks in the mass spectrum, and no top squark coannihilation region appears. Instead, new allowed regions appear: the vertical band near $\alpha \sim 6$ corresponds to an $A$-funnel annihilation region[27], where $2 m_{\widetilde{Z}_{1}} \simeq m_{A}$. The upper part of the $A$-funnel is somewhat beyond the $100 \mathrm{fb}^{-1}$ LHC reach. In addition, the upper part of the vertical band near $\alpha \sim 4$ occurs where the value of $|\mu|$ drops, and the $\widetilde{Z}_{1}$ becomes mixed higgsino-bino dark matter (MHDM). This is due in part to the relatively low value of $\left|M_{3}\right|$ at $M_{\mathrm{GUT}}$, which feeds through the RGEs to yield a low $\mu$ value (see Ref. [28, 29] for a discussion of the low $\left|M_{3}\right|$ dark matter model). The MHDM has a large annihilation cross section to $W^{+} W^{-}$and $Z Z$, which acts to reduce the relic density, as in the focus point region of the mSUGRA model. The broad allowed region at low $m_{3 / 2}$ is where $\mu \sim M_{1} \sim M_{2}$, and so here we actually find mixed bino-wino-higgsino dark matter [30]. In this region, LHC collider events should be rich in $b$ and $\tau$ jets, since $\tilde{t}_{1}$ is produced in many cascade decays and $\tilde{t}_{1} \rightarrow b \widetilde{W}_{1}$, while $\widetilde{Z}_{2}$ also gets produced, followed by $\widetilde{Z}_{2} \rightarrow \tilde{\tau}_{1} \bar{\tau}+\bar{\tau}_{1} \tau$. A sample point with a mixed bino-wino-higgsino LSP is shown as Point 1 in Table 1. This point would likely be excluded due to $B F(b \rightarrow s \gamma)$ constraints (see Sec. 3 ) and possibly by direct dark matter search constraints (see Sec. 5). The BWCA region at $\alpha \sim-2$ is also present in this frame.

In frame $d$ ), with the same modular weights as $c$ ) but with $\tan \beta=30$, the overall picture is similar to $c$ ) for $0<\alpha \lesssim 5$, but the Higgs funnel disappears into the stau LSP forbidden region. The entire WMAP allowed region is accessible to the LHC. For $\alpha<0$, the BWCA region present for the $\tan \beta=10$ case disappears, leaving no WMAP-allowed region.

\footnotetext{
${ }^{4}$ While the LHC reach was calculated in Ref. [25] for the case of the mSUGRA model, it was found that the reach mainly depends on the squark and gluino masses, and not on their particular decay modes, so long as there is ample energy release in the squark and gluino cascade decays. For this reason, we expect that the reach results of Ref. [25] will also apply approximately to the case of MM-AMSB models, the exception being where the energy released in the primary decays is suppressed for kinematic reasons [16].
} 


\begin{tabular}{|c|c|c|c|c|}
\hline parameter & Point 1 & Point 2 & Point 3 & Point 4 \\
\hline$\left(n_{H}, n_{m}\right)$ & $\left(0, \frac{1}{2}\right)$ & $(0,1)$ & $(0,1)$ & $(0,1)$ \\
\hline$\alpha$ & 4 & 7.5 & -10 & -10 \\
\hline$m_{3 / 2}(\mathrm{TeV})$ & 10.0 & 60.0 & 4.0 & 4.0 \\
\hline $\tan \beta$ & 10 & 10 & 10 & 10 \\
\hline$\mu$ & 161.9 & 490.0 & 416.4 & -416.3 \\
\hline$m_{\tilde{g}}$ & 393.6 & 4699.1 & 701.7 & 701.0 \\
\hline$m_{\tilde{u}_{L}}$ & 354.2 & 3979.7 & 652.9 & 652.5 \\
\hline$m_{\tilde{t}_{1}}$ & 127.7 & 2631.5 & 436.8 & 420.8 \\
\hline$m_{\tilde{b}_{1}}$ & 312.7 & 3531.2 & 582.4 & 584.0 \\
\hline$m_{\tilde{e}_{L}}$ & 210.7 & 1493.0 & 198.1 & 198.0 \\
\hline$m_{\tilde{e}_{R}}$ & 191.0 & 837.5 & 108.9 & 108.8 \\
\hline$m_{\tilde{\tau}_{1}}$ & 183.0 & 800.7 & 97.6 & 92.3 \\
\hline$m_{\widetilde{W}_{1}}$ & 131.6 & 513.9 & 185.8 & 178.1 \\
\hline$m_{\widetilde{Z}_{2}}$ & 169.5 & 502.8 & 185.5 & 178.1 \\
\hline$m_{\widetilde{Z}_{1}}$ & 118.5 & 499.3 & 66.8 & 64.9 \\
\hline$m_{A}$ & 305.5 & 3236.7 & 510.1 & 515.5 \\
\hline$m_{h}$ & 110.7 & 124.2 & 111.4 & 113.0 \\
\hline$\Omega_{\widetilde{Z}_{1}} h^{2}$ & 0.001 & 0.04 & 0.11 & 0.11 \\
\hline$B F(b \rightarrow s \gamma)$ & $2.9 \times 10^{-5}$ & $3.3 \times 10^{-4}$ & $5.4 \times 10^{-4}$ & $2.9 \times 10^{-4}$ \\
\hline$\Delta a_{\mu}$ & $34.0 \times 10^{-10}$ & $0.5 \times 10^{-10}$ & $-39.4 \times 10^{-10}$ & $41.6 \times 10^{-10}$ \\
\hline$B F\left(B_{s} \rightarrow \mu^{+} \mu^{-}\right)$ & $4.4 \times 10^{-9}$ & $3.8 \times 10^{-9}$ & $3.7 \times 10^{-9}$ & $4.1 \times 10^{-9}$ \\
\hline$\sigma_{s c}\left(\widetilde{Z}_{1} p\right)$ & $3.1 \times 10^{-7} \mathrm{pb}$ & $3.9 \times 10^{-10} \mathrm{pb}$ & $1.9 \times 10^{-10} \mathrm{pb}$ & $8.9 \times 10^{-10} \mathrm{pb}$ \\
\hline
\end{tabular}

Table 1: Masses and parameters in GeV units for four cases of the MM-AMSB model. Also shown are predictions for low energy observables, together with the cross section for direct detection of dark matter. In all cases, we take $m_{t}=175 \mathrm{GeV}$.

In frame $e$ ), we show results for modular weights $n_{H}=0, n_{m}=1$ with $\tan \beta=10$. Since $n_{m}=1$, in Eq. (1.4) the leading contribution proportional to $\alpha^{2}$ for matter scalars is absent, and so squark and slepton masses are suppressed, and usually we find tachyonic or charged LSPs in parameter space. A narrow region around $\alpha \sim 5-8$ survives where $m_{\widetilde{Z}_{1}} \sim m_{\tilde{\tau}_{1}}$, while the $\widetilde{Z}_{1}$ is a nearly pure higgsino state. This region of parameter space has tachyonic slepton masses at the GUT scale. The upper portion of this region is in accord with WMAP/LEP2 constraints, but is beyond the reach of both the LHC and ILC, but may be accessible to ton-size dark matter detectors. A sample point for $\alpha=7.5$ and $m_{3 / 2}=60 \mathrm{TeV}$ is shown as Point 2 in Table 1 . There are also some faint regions that survive for negative $\alpha$ values; the WMAP/LEP2-allowed portion of these has sleptons of mass $\sim 100 \mathrm{GeV}$, which leads to so-called bulk annihilation of neutralinos through light slepton exchange. A sample bulk annihilation point is listed as Point 3 in Table 1. While this point is WMAP/LEP2 allowed, it has a large negative contribution to $(g-2)_{\mu}$ (see Sec. 3), and is likely excluded. By flipping the sign of $\mu$, the $(g-2)_{\mu}$ contribution flips sign, and gives a large positive contribution: see Point 4 of Table 1 . In frame f), no parameter 
space points lead to a viable SUSY spectrum. This is due to a combination of tachyonic GUT scale soft masses plus the downward push of a large $\tau$ Yukawa coupling, such that weak scale tachyonic masses are produced all over parameter space.

\section{$2.2 n_{H}=1 / 2$ cases}

In Fig. 2, we present an overview of the MM-AMSB parameter space for $n_{H}=1 / 2$ and various $n_{m}$ possibilities, for $\tan \beta=10$ and 30 , with $\mu>0$. Frame $a$ ) shows the $\alpha$ vs. $m_{3 / 2}$ plane for $n_{m}=0$ and $\tan \beta=10$. The shading and labelling is as in Fig. 1. We see once again that a region has appeared where the $\tilde{t}_{1}$ is the LSP, although this region is smaller than in Fig. 1 1 a). The reason again is that the $A_{t}$ parameter is quite large (reduced only by the $n_{H}=1 / 2$ factor in Eq. (1.3) ) at the GUT scale, and again this serves to reduce the top squark soft masses via RG evolution. The red-shaded region to the right of the turquoiseforbidden region again occurs due to top-squark co-annihilation effects. Along the region of high $m_{3 / 2}$ and $\alpha \sim 4, \tilde{g} \tilde{g}$ will typically be followed by $\tilde{g} \rightarrow t \tilde{t}_{1}$, with $\tilde{t}_{1} \rightarrow c \widetilde{Z}_{1}$, so that gluino pair events at the LHC will be characterized by the presence of a pair of top quarks plus two soft charm jets and additional $E_{T}^{\text {miss }}$. The region at $\alpha \sim 2.5$ is again a region with a $\widetilde{Z}_{1}$ that is a mixed bino-wino-higgsino state. There also appears an $A$-annihilation funnel at $\alpha \sim 5$, and a small BWCA region at $\alpha \sim-2$. The picture for $\tan \beta=30$ is illustrated in frame $b$ ), where the tau slepton becomes lighter due to the effect of the large tau-lepton Yukawa coupling. In this case, the base of the $A$-funnel gets wider due to the addition of stau-neutralino co-annihilation. In addition, a region of bulk annihilation through light staus opens up at negative $\alpha$.

In frame $c$ ), we see results for $n_{m}=1 / 2$ and $\tan \beta=10$. The stop-LSP forbidden region has disappeared owing to the increased matter modular weights. Now there exists a broad region around $\alpha \sim 3-4$ with a mixed higgsino-bino $\widetilde{Z}_{1}$, while the low $m_{3 / 2}$ part includes an LSP with a significant wino component. The $A$-annihilation funnel is present at $\alpha \sim 6-7$, and a BWCA region occurs at $\alpha \sim-2$. If we shift to large $\tan \beta=30$ in frame $d$ ), then a large excluded stau-LSP region occurs. To the right of this region occurs an area of mixed $A$-funnel/stau co-annihilation, while the region to the left is characterized by a higgsino-LSP with some degree of stau co-annihilation. We note here that some part of the $A$-annihilation funnel extends out beyond the reach of LHC with $100 \mathrm{fb}^{-1}$; in this region, $m_{\tilde{g}} \sim m_{\tilde{q}} \sim 3.5 \mathrm{TeV}$. Any WMAP-allowed regions at negative $\alpha$ have disappeared.

For $n_{m}=1$ and $\tan \beta=10$, portrayed in frame $e$ ), almost all allowed regions have disappeared. There are a few allowed points to the left of the excluded region at positive $\alpha$, plus a small region of bulk annihilation via light sleptons with mass $\sim 100 \mathrm{GeV}$ at $\alpha<-5$. If we increase $\tan \beta$ to 30 as in frame $f$ ), then only a few points persist at positive $\alpha$, while a small band around $\alpha \sim-6$ exists for negative $\alpha$ which is characterized by a bino-like LSP and stau co-annihilation.

\section{$2.3 \mathrm{n}_{\mathrm{H}}=1$ cases}

In Fig. 3, we show the parameter space regions for Higgs modular weight $n_{H}=1$, and various matter modular weights with $\tan \beta=10$ and 30 . In frame $a$ ), with $n_{m}=0$ and $\tan \beta=10$, it is noteworthy that the stop-LSP region which is present in Figs. 1 $1 a$ ) and $2 a$ ) 
has disappeared, even though the matter modular weight remains the same. In this case, just as in the $n_{m}=1 / 2, n_{H}=0$ case in Fig. 1 $c$, the large Higgs modular weight reduces the value of $A_{t}\left(M_{G U T}\right)$ enough so that the top squark soft masses are not driven to such low values. The large Higgs modular weight also reduces the GUT scale Higgs mass, which also reduces the weak scale $A$ mass[31]. Thus, the $A$ funnel region moves out to somewhat higher values of $\alpha$ than found in Fig. 2 $a$ a). There also remains a large region of higgsino and mixed higgsino-wino-bino dark matter around $\alpha \sim 3$. The BWCA region maintains a presence at small, negative $\alpha$ values. The CERN LHC can cover all the parameter space shown. Moving to $\tan \beta=30$ in frame $b$ ), we find the base of the $A$-funnel widened somewhat compared to frame $a$ ) due to the presence of stau co-annihilation. In addition, a region has appeared at low $m_{3 / 2}$ and negative $\alpha$ where neutralino bulk annihilation through light staus can occur.

In frame $c$ ), we plot parameter space for $n_{H}=1, n_{m}=1 / 2$, the same choices adopted in the non-zero modular weight analysis of Ref. [13]. Here, we have an $A$-annihilation region at large $\alpha$, a higgsino region at small positive $\alpha$, and a BWCA region at small negative $\alpha$. In this case, the LHC covers the entire $A$-funnel, unlike Figs. 1 $c$ ) and $2 c$ ). In frame $d$ ) for $\tan \beta=30$, a large stau-LSP excluded region has appeared. In addition, the $A$-funnel has moved to very large $\alpha$ values, and is very broad, owing to an overlap with the stau co-annihilation region.

Moving to $n_{H}=1$ and $n_{m}=1$ portrayed in frame $e$ ) for $\tan \beta=10$, we see that all the $\alpha>0$ region is now excluded due to a stau LSP. In this case, the moduli contribution to scalar masses only comes from the mixing term in Eq. 1.4, and so the scalar spectrum is similar to AMSB, where sleptons have tachyonic masses. For negative $\alpha$ values, the AMSB-moduli interference term in Eq. 1.4 becomes positive, so some spectra are allowed. The only WMAP-allowed points occur at very low $m_{3 / 2}$ values, and are typified by bulk neutralino annihilation through light sleptons. In the case of large $\tan \beta=30$ shown in frame $f$ ), again, no allowed regions appear for $\alpha>0$, while some points at large negative $\alpha$ are allowed where neutralino annihlation occurs through light slepton exchange in the $t$-channel. This region, we will see, turns out to be largely excluded because it yields a large, negative value of $(g-2)_{\mu}$, in contradiction with experimental measurements whose discussion we now turn to.

\section{3. $(\mathrm{g}-2)_{\mu}$ and $\mathrm{BF}(\mathrm{b} \rightarrow \mathrm{s} \gamma)$ in the MM-AMSB model}

\section{$3.1(\mathrm{~g}-2)_{\mu}$}

Current measurements of the muon anomalous magnetic moment show an apparent deviation from SM predictions. Combining QED, electroweak, hadronic (using $e^{+} e^{-} \rightarrow$ hadrons to evaluate hadronic loop contributions) and light-by-light contributions, and comparing against measurements from E821 32] at BNL, a positive deviation in $a_{\mu} \equiv \frac{(g-2)_{\mu}}{2}$ of

$$
\Delta a_{\mu}=a_{\mu}^{e x p}-a_{\mu}^{S M}=22(10) \times 10^{-10}
$$


is reported in the Particle Data Book 33], i.e. a 2.2 $\sigma$ effect. Within this framework, corrections from Standard Model expectations arise from one loop diagrams with either $\widetilde{W}_{i}-\tilde{\nu}_{\mu}$ or $\widetilde{Z}_{i}-\tilde{\mu}_{1,2}$ in the loop, possibly accounting for the (rather weak) discrepancy.

In Fig. [1 we show $a_{\mu}^{\mathrm{SUSY}}$ [34], the SUSY contribution versus $m_{\tilde{\mu}_{L}}$ arising from the various modular weight choices in the MM-AMSB model. Only points with $\Omega_{\widetilde{Z}_{1}} h^{2}<0.13$ are plotted in this figure. The various colors/shapes of the plotting symbols correspond to different choices of modular weights, as listed on the figure. We see from Fig. (Ga) and Fig. $⿴ b$ ) that, as expected, the SUSY contributions from models with large $m_{\tilde{\mu}_{L}}$ (and concomitantly large $m_{\tilde{\nu}_{\mu}}$ and $m_{\tilde{\mu}_{R}}$ ) give almost no contributions, since the contributions are suppressed by the large sparticle masses. However, depending on the value of $\tan \beta$, models with $m_{\tilde{\mu}_{L}}$ ranging from about $200 \mathrm{GeV}$ to several hundred $\mathrm{GeV}$ can give sizable contributions, and even accommodate the theory-experiment deviation. For the larger $\tan \beta$ case, very light sleptons in fact give too large a SUSY contribution to the muon magnetic moment. In addition, several sets of models give large negative contributions to $a_{\mu}^{\text {SUSY }}$. These are the models with $n_{m}=1$ and any choice of $n_{H}$ but with moderate to large values of negative $\alpha$, wherein matter scalar masses are suppressed, and the points are WMAP-allowed by bulk annihilation of neutralinos via light $t$-channel slepton exchange. Even though we take $\mu>0$ in these models, we know that

$$
a_{\mu}^{\mathrm{SUSY}} \sim \frac{m_{\mu}^{2} \mu M_{i} \tan \beta}{M_{\mathrm{SUSY}}^{4}}
$$

and since the weak scale gaugino masses $M_{1,2}$ are usually negative in these cases, a negative contribution to $a_{\mu}^{\mathrm{SUSY}}$ arises. Thus (even allowing for considerable theoretical uncertainties) these models with $a_{\mu}^{\text {SUSY }} \sim-40$ are likely ruled out.

The situation is more clearly illustrated in Fig. 5, where we plot $a_{\mu}^{\mathrm{SUSY}}$ versus $\alpha$ for a) $\tan \beta=10$ and $b) \tan \beta=30$. We see that models with $\alpha<-5$ give large negative contributions to $a_{\mu}^{\mathrm{SUSY}}$. Thus, when $\alpha \lesssim-5$, models with $\mu<0$ should give $\Delta a_{\mu}$ allowed regions. Models where agreement with the measured CDM relic density is obtained via BWCA (these do not occur for the $\tan \beta=30$ cases) yield a smaller value of $a_{\mu}^{\text {SUSY }}$, in part because the sleptons are heavier, and in part because of the relative sign between $M_{1}$ and $M_{2}$ that leads to a negative interference between the chargino and neutralino loop diagrams.

\section{$3.2 \mathrm{BF}(\mathrm{b} \rightarrow \mathrm{s} \gamma)$}

The branching fraction $B F(b \rightarrow s \gamma)$ serves as a strong constraint on SUSY models in part because, if $m_{\mathrm{SUSY}} \simeq M_{W}$, supersymmetric contributions mediated by $\widetilde{W}_{i} \tilde{t}_{j}$ and $b H^{+}$ loops are expected to occur at similar rates 35] to the SM contribution, mediated by a $t W$ loop. The measured branching fraction- from a combination of CLEO, Belle and BABAR experiments[36] - is $B F(b \rightarrow s \gamma)=(3.55 \pm 0.26) \times 10^{-4}$, while the latest SM calculations find[37] $B F(b \rightarrow s \gamma)=(3.29 \pm 0.33) \times 10^{-4}$. Thus, any SUSY contribution to $B F(b \rightarrow s \gamma)$ seems highly suppressed.

The results from the MM-AMSB models for various modular weight choices and with $\Omega_{\widetilde{Z}_{1}} h^{2}<0.13$ are shown in Fig. 6, where we plot $B F(b \rightarrow s \gamma)$ vs. $m_{\tilde{g}}$ for $\left.a\right) \tan \beta=10$ and 
b) $\tan \beta=30$. Many of the MM-AMSB models with $m_{\tilde{g}} \lesssim 1000 \mathrm{GeV}$ predict $B F(b \rightarrow s \gamma)<$ $2 \times 10^{-4}$, and thus are likely ruled out (unless small flavor-changing contributions to squark mass matrices are invoked). Since $m_{\tilde{g}}$ is strongly correlated with $m_{\tilde{q}}$ (see Fig. 7 below), these are models with relatively light squarks (and sleptons). Models with $\alpha<0$ that are in agreement with the measured CDM relic density due to bulk annihilation via very light sleptons (and correspondingly light squarks) typically give too high a prediction for $B F(b \rightarrow s \gamma)$, and are again likely ruled out. In frame $b)$ with $\tan \beta=30$, the contributions to $B F(b \rightarrow s \gamma)$ can be even more anomalous, and a much larger fraction of models with $m_{\tilde{g}} \lesssim 1000 \mathrm{GeV}$ are likely ruled out.

\section{Collider searches in the MM-AMSB Model}

\subsection{Fermilab Tevatron $p \bar{p}$ collider}

It is possible to search for gluino and squark pair production at the Fermilab Tevatron $p \bar{p}$ collider by looking for multi-jet $+E_{T}^{\text {miss }}$ signals. While many Tevatron $E_{T}^{\text {miss }}+$ jets searches for models with gaugino mass universality have been pre-empted by LEP2 searches for chargino pair production, in models with non-universal gaugino masses, gluino and squark searches may still be of interest. Indeed, in the so-called "low $M_{3}$ dark matter model" (LM3DM) 28, the Tevatron with $5 \mathrm{fb}^{-1}$ of integrated luminosity was found to be sensitive to $m_{\tilde{g}} \sim 200-350 \mathrm{GeV}$ in models beyond the reach of LEP2[29]. The MM-AMSB models share with LM3DM models the characteristic that $M_{3}$ is reduced relative to $M_{1}$ and $M_{2}$ at the GUT scale, since the AMSB contribution to $M_{3}$ subtracts from the moduli-mediated contribution.

In Fig. 8, we plot $m_{\tilde{u}_{R}}$ vs. $m_{\tilde{g}}$ for MM-AMSB models with $\left.a\right) \tan \beta=10$ and $b$ ) $\tan \beta=30$, and all modular weight choices, where we require $\Omega_{\widetilde{Z}_{1}} h^{2}<0.13$ and $m_{\widetilde{W}_{1}}>103$ $\mathrm{GeV}$. The diagonal dashed line denotes where $m_{\tilde{u}_{R}}=m_{\tilde{g}}$. One lesson from this plot is that generic first and second generation squark masses are comparable in mass to $m_{\tilde{g}}$ for all modular weight choices; the possibility that $m_{\tilde{q}} \gg m_{\tilde{g}}$ - which occurs in the large $m_{0}$ region of the mSUGRA model- does not occur here. We also find that in some cases (such as $n_{H}=1, n_{m}=0$ ) the value of $m_{\tilde{g}} \sim m_{\tilde{q}}$ can be as low as $\sim 300 \mathrm{GeV}$, and hence may be amenable to Tevatron gluino and squark searches, where the current limit for $m_{\tilde{q}} \simeq m_{\tilde{g}}$ is $\sim 325 \mathrm{GeV}$ within the mSUGRA framework 38].

It is also possible for Tevatron experiments to search for SUSY via $p \bar{p} \rightarrow \widetilde{W}_{1}^{ \pm} \widetilde{Z}_{2} X \rightarrow$ $3 \ell+E_{T}^{\text {miss }}+X$ events [39]. The clean trilepton search is viable when the "spoiler" decay modes $\widetilde{Z}_{2} \rightarrow \widetilde{Z}_{1} h$ and $\widetilde{Z}_{2} \rightarrow \widetilde{Z}_{1} Z$ are closed [40], i.e. when $m_{\widetilde{Z}_{2}}-m_{\widetilde{Z}_{1}}<M_{Z}$ and $m_{h}$. We plot in Fig. 8 the $m_{\widetilde{Z}_{2}}-m_{\widetilde{Z}_{1}}$ mass difference versus $m_{\widetilde{W}_{1}}$ for all modular weight choices and WMAP/LEP2-allowed models, for $a) \tan \beta=10$ and $b) \tan \beta=30$. The region above the horizontal dashed line is where the spoiler decay $\widetilde{Z}_{2} \rightarrow \widetilde{Z}_{1} Z$ turns on. We see a large fraction of MM-AMSB models of all modular weights are below this line, and thus have closed spoiler modes, so that $\widetilde{Z}_{2} \rightarrow \widetilde{Z}_{1} \ell \bar{\ell}$ is likely to have a significant branching fraction (an exception occur if interference effects suppress the leptonic branching decay or if the decay $\widetilde{Z}_{2} \rightarrow \tau \tilde{\tau}_{1}$ becomes accessible). Depending on $B F\left(\widetilde{Z}_{2} \rightarrow \widetilde{Z}_{1} \ell \bar{\ell}\right)$, a portion of the lower 
range of $m_{\widetilde{W}_{1}} \sim 100-200 \mathrm{GeV}$ and $m_{\widetilde{Z}_{2}}-m_{\widetilde{Z}_{1}}<M_{Z}$ may thus be accessible to Tevatron clean trilepton searches.

Finally, in Fig. 9 we plot the values of $m_{h}$ vs. $m_{\tilde{t}_{1}}$ for WMAP/LEP2-allowed MMAMSB models and all modular weight choices. Here, we see that $m_{h} \lesssim 125-130 \mathrm{GeV}$, so that Tevatron Higgs searches may see evidence of $W h, Z h$ production depending on the integrated luminosity achieved. In addition, the $\tilde{t}_{1}$ mass can range down to $\sim 100 \mathrm{GeV}$ in MM-AMSB models and may be accessible to Tevatron searches 41. However, in these cases, the $m_{\tilde{t}_{1}}-m_{\widetilde{Z}_{1}}$ mass gap is usually quite small, in which case the $\tilde{t}_{1}$ decay products will be soft, making hadron collider searches more difficult.

We note here also that in the MM-AMSB models with $m_{\tilde{t}_{1}}<m_{t}$ and $m_{h}$ is light, some of the conditions for successful electroweak baryogenesis are satisfied. However, in Ref. [42], it is noted that the soft term $m_{\tilde{t}_{R}}^{2}<0$ condition also ought to be satisfied. We find that in MM-AMSB models $m_{\tilde{t}_{R}}^{2}>0$ and the light $\tilde{t}_{1}$ arises in part due to the large $A_{t}$ term; this apparently contradicts the requirements for successful EW baryogenesis in these models. Of course, this would be moot unless there is also a significant $C P$ violating phase $(\gtrsim 0.05)$ either via the $b$-term, or via corrections to other SSB parameters.

\subsection{CERN LHC $p p$ collider}

The CERN LHC $p p$ collider will begin data taking in earnest in 2008 running at $\sqrt{s}=14$ $\mathrm{TeV}$. The search for supersymmetry by Atlas and CMS will initially focus on gluino and squark production followed by cascade decays. It has been shown in Ref. 25] that in the case where $m_{\tilde{q}} \sim m_{\tilde{g}}$ as in MM-AMSB models, LHC experiments should have a reach to

$m_{\tilde{g}} \lesssim 3 \mathrm{TeV}$ for an integrated luminosity of $100 \mathrm{fb}^{-1}$. Except for possibly cases where the sparticle masses are all roughly degenerate (where $\mu_{\text {mir }} \sim 1 \mathrm{TeV}$ ) so that the visible decay products are significantly softer than the expectation in the mSUGRA model 16], we would expect a similar reach within the MM-AMSB framework. We can see from Fig. 7 that most MM-AMSB models should be detectable at CERN LHC. Exceptions occur in the cases of $n_{H}=0, n_{m}=1$ models for $\tan \beta=10$, and $n_{H}=1 / 2, n_{m}=1$ models for $\tan \beta=30$. In these instances, the thin allowed region to the left of the excluded region for $\alpha \sim 5-7$ gives rise to cases with $m_{\tilde{g}}$ ranging up to $4-5 \mathrm{TeV}$, which is well-past the LHC reach for any projected integrated luminosity value.

Fig. 8 shows also that a large fraction of models contain a mass difference $m_{\widetilde{Z}_{2}}-m_{\widetilde{Z}_{1}}<$ $M_{Z}$. Thus, decays of $\widetilde{Z}_{2}$ s produced mostly via cascade decays of gluinos and squarks, should lead to a measurable dilepton mass edge with $m(\ell \bar{\ell})$ bounded by $m_{\widetilde{Z}_{2}}-m_{\widetilde{Z}_{1}}$ unless the branching fraction for the decay is strongly suppressed. This mass edge can serve as a starting point for gluino and squark cascade decay reconstruction 43.

\subsection{Linear $e^{+} e^{-}$collider}

Experiments at a linear $e^{+} e^{-}$collider will most easily be able to discover charginos, and sleptons (including sneutrinos, if these decay visibly) via their pair production. Moreover, unless the mass gaps $m_{\widetilde{W}_{1}}-m_{\widetilde{Z}_{1}}$ or $m_{\tilde{\ell}}-m_{\widetilde{Z}_{1}}$ are very small, signals from their production should be readily visible over Standard Model backgrounds essentially all the way to the 
kinematic limit for their production. Specialized analyses allow the signal to be extracted for smaller values of the sparticle-LSP mass gap [26]. We show in Fig. 10 the $\tilde{\tau}_{1}$ mass versus $\widetilde{W}_{1}$ mass for all MM-AMSB models consistent with WMAP/LEP2. A $\sqrt{s}=500$ $\mathrm{GeV}$ machine should have a reach to $m_{\widetilde{W}_{1}}$ or $m_{\tilde{\tau}_{1}} \sim 250 \mathrm{GeV}$, while a $\sqrt{s}=1000 \mathrm{GeV}$ machine will have a reach to $m_{\widetilde{W}_{1}}$ or $m_{\tilde{\tau}_{1}} \sim 500 \mathrm{GeV}$, as indicated by the dashed reach lines. Thus, while the ILC would certainly see the light Higgs boson $h$ in all MM-AMSB cases, it would only be able to access sparticles for a fraction of the models plotted.

\section{Dark matter searches}

In addition to collider searches for sparticles and Higgs bosons, searches for dark matter relics from the Big Bang, either via direct detection experiments at underground dark matter detectors, or indirectly via searches for neutralino annihilation to high energy neutrinos in the core of the sun, or neutralino annihilations to high energy gamma rays or antimatter in the galactic halo, may provide an independent signal for new physics. The results of these DM searches are sensitive to the composition of the LSP, and combining these with information from collider experiments may help us to zero in on the underlying SUSY model.

\subsection{Direct dark matter searches}

In the case of particle physics models with $R$-parity conserving supersymmetry and a neutralino LSP, dark matter detectors look for rare neutralino-nucleus collisions in experiments located deep underground, where the neutralino is expected to deposit of order ten $\mathrm{keV}$ of energy in an elastic scattering event. The neutralino-nucleus scattering cross section naturally breaks up into spin-independent (SI) and spin-dependent (SD) pieces, where at present the greatest experimental sensitivity is to SI cross sections, since in this case the neutralino couples coherently to the entire nucleus, so that the cross section increases as $A^{2}$, where $A$ is the mass number of the nucleus. To facilitate comparision between different detector materials, the spin-independent scattering cross section off a single proton is usually used as a figure of merit. We use the IsaRes code (part of the Isajet/Isatools package 20, 44]) to evaluate this associated direct dark matter detection cross section in MM-AMSB models.

In Fig. 11, we plot the expectation for $\sigma_{S I}\left(\widetilde{Z}_{1} p\right)$ as a function of $m_{\widetilde{Z}_{1}}$ for all nine sets of modular weights, retaining only those points consistent with constraints from both WMAP and LEP2 data for $a) \tan \beta=10$, and $b) \tan \beta=30$. Presently, the most stringent limit on this cross section comes from the CDMS collaboration 45], and is shown by the top-most contours labelled CDMS. Since the neutralino can transfer the maximum energy to the nucleus if its mass is $\sim$ the mass of the nuclear target $m_{T}$, the sensitivity is the greatest when $m_{\widetilde{Z}_{1}} \sim m_{T}$. The maximum energy that can be transferred reduces rapidly if $m_{\widetilde{Z}_{1}} \ll m_{T}$, and saturates if the neutralino is very heavy, accounting for the general shape of this contour (as well as of the projected reach contours discussed below). We see that, especially for the larger value of $\tan \beta$, the CDMS experiment has already excluded neutralinos as heavy as $300-400 \mathrm{GeV}$ for some choices of modular weights. CDMS II is now 
operating, and is expected to reach cross sections at the $10^{-8} \mathrm{pb}$ level which will begin probing MM-AMSB parameter space with neutralinos as heavy as 500-800 GeV depending on $\tan \beta$. CDMS plans an upgrade to 7 supertowers located in the Sudbury underground site, where a sensitivity to cross sections at the $10^{-9} \mathrm{pb}$ level is anticipated 446]. To go further, ton-scale noble liquid detectors will have to be used. As an example, we plot the reach of the proposed Warm Argon Project (WARP) $1400 \mathrm{~kg}$ detector [47, which will endeavour to probe cross sections as low as $10^{-10} \mathrm{pb}$. We see that if proposed ton-sized noble gas detectors do achieve the projected sensitivity, they should be able to probe the bulk of MM-AMSB parameter space unless $\widetilde{Z}_{1}$ is rather heavy. Exceptions where light

neutralinos $\left(m_{\widetilde{Z}_{1}} \lesssim 500 \mathrm{GeV}\right)$ would escape detection even at these facilities occur when the neutralino is essentially bino-like, and the WMAP relic density is attained via squark or slepton co-annihilation, or via BWCA.

\subsection{Indirect dark matter searches}

In addition to direct detection experiments discussed above, there are also indirect searches for dark matter that rely on the detection of a flux of high energy particles, or of antiparticles, produced via the annihilation of neutralinos in our galactic halo 48.

The IceCube [9] and ANTARES [50] experiments are designed to detect high energy neutrinos from the annihilation of neutralinos gravitationally trapped in the core of the Sun[51]. In Fig. 12, we present rates for detection of $\nu_{\mu} \rightarrow \mu$ conversions in the Antarctic ice, with the muons being detected in the IceCube experiment. We show results for $a) \tan \beta=$ 10 , and $b) \tan \beta=30$. The reference experimental sensitivity that we show is for a flux of 40 muons per $\mathrm{km}^{2}$ per year with $E_{\mu}>50 \mathrm{GeV}$. We see that while many sets of modular weights lead to a potentially detectable signal for neutralinos as heavy as $1 \mathrm{TeV}$ (e.g. $\left.n_{H}=1, n_{m}=0\right)$, there are other models where the signal is always below the observable level (e.g. $\left.n_{H}=0, n_{m}=0\right)$. This may be understood if we recognize that the signal at IceCube will be largely governed by the higgsino content of the neutralino since this controls the neutralino-nucleon scattering cross section (essential for trapping the neutralinos in the first place) and, along with the wino content, also affects the neutralino annihilation rate. Thus, when we can only have a bino LSP (as, for instance, for $n_{H}=n_{m}=0$ case) the signal will be small. In contrast, regions with mixed higgsino or mixed wino-higgsino-bino DM will typically lead to the largest signals at IceCube.

Neutralinos in our galactic halo may also annihilate into anti-particles or anti-nuclei, thus providing new ways for their indirect detection, though signals from neutrinos not pointing from the sun are below their background. Positron detection experiments include HEAT [52], Pamela [53] and AMS-02 [54; antiprotons may be detected by BESS [55], Pamela, AMS-02; anti-deuterons by BESS[56], AMS-02 and GAPS[57]. For positrons and anti-protons we evaluate the averaged differential antiparticle flux in a projected energy bin centered at a kinetic energy of $20 \mathrm{GeV}$, where we expect an optimal statistics and signal-to-background ratio at space-borne antiparticle detectors [58]. We take the experimental sensitivity to be that of the Pamela experiment after three years of data-taking as our benchmark. Finally, the average differential anti-deuteron flux has been computed in the $0.1<T_{\bar{D}}<0.25 \mathrm{GeV}$ range, where $T_{\bar{D}}$ stands for the antideuteron kinetic energy 
per nucleon, and compared to the estimated GAPS sensitivity for an ultra-long duration balloon-borne experiment [57].

Our projection for the sensitivity of Pamela $\left(\bar{p}\right.$ and $\left.e^{+}\right)$and GAPS experiments $(\bar{D})$ is shown in Fig. 13. We show these for $\tan \beta=10$ (left column) and $\tan \beta=30$ (right column). The expected fluxes depend on the (unknown) details of the neutralino distribution in our galactic halo, and are shown for the Burkert profile [59] obtained if it is assumed that the central cusp of the DM halo seen in numerical simulations is smoothed out by heating of colder particles. This less clumpy halo distribution yields lower fluxes for the anti-particles so that our projections for the reach of these indirect search experiments may be regarded as conservative.

We see that typically only a small fraction of the models will be within the reach of Pamela, with a somewhat larger fraction within the reach of GAPS. Perhaps, more perti-

nently, most models with $m_{\widetilde{Z}_{1}} \gtrsim 300-400 \mathrm{GeV}$ are projected to be beyond the reach of these experiments. We should mention, however, that this conclusion is sensitive to our assumption of the Burkert halo profile. If instead we assume a scenario where baryon infall causes progressive deepening of the gravitational potential well, a considerably clumpier halo distribution is obtained. For the resulting Adiabatically Contracted N03 Halo Model [60], we have checked that we get larger fluxes by factors of $\sim 15-20$ (anti-protons), $\sim 5-7.5$ (positrons) and $\sim 10-15$ (anti-deuterons) so that the reach is correspondingly increased.

Indirect detection of neutralinos is also possible via the detection of high energy gamma rays [61] from produced by neutralino annihilation in the centre of our Galaxy [62]. These will also be searched for by the GLAST collaboration 63]. We have evaluated expectations for the integrated continuum $\gamma$ ray flux above a $E_{\gamma}=1 \mathrm{GeV}$ threshold in the MM-AMSB model. Since these are extremely sensitive to the assumed neutralino halo distribution, we show in Fig. 14 results for both the Burkert profile (upper frames) and the Adiabatically Contracted N03 Halo Model (lower frames), again for $\tan \beta=10$ (left frames) and $\tan \beta=30$ (right frames). The horizontal line marks the expected sensitivity of GLAST, $1.0 \times 10^{-10} \mathrm{~cm}^{-2} \mathrm{~s}^{-1}$. We see that while it almost appears that the entire MM-AMSB parameter space will be accessible to GLAST for the optimistic projection using the N03 profile, the inference using the Burkert profile is completely different. We need to better understand the halo profile before any definitive statement can be made, but at least for some models, the reach of GLAST even very conservatively extends out to $m_{\widetilde{Z}_{1}}=500 \mathrm{GeV}$.

\section{Testing mirage unification for $\alpha<0$ and very large $\mu_{\text {mir }}$}

The distinguishing characteristic of MM-AMSB models is that the SSB gaugino mass parameters, when extrapolated to high scales using one loop RGEs are expected to exhibit unification at the scale $Q=\mu_{\text {mir }}$ given by Eq. (1.6). We emphasize that there is no physical threshold at this scale and the phenomenon is therefore referred to as mirage unification. For positive values of $\alpha, M_{\text {weak }} \leq \mu_{\text {mir }} \leq M_{\mathrm{GUT}}$, and it is straightforward to test the mirage unification of gaugino mass parameters since the weak scale SSB gaugino masses can be extracted from measurements at the proposed electron-positron linear collider. By evolving the corresponding weak scale sfermion SSB parameters from the weak scale, the 
expected concomitant mirage unification of first generation scalar masses- truly a smoking gun confirmation of the scenario- may also be revealed [17]. An example of the evolution of gaugino mass parameters evolved from $M_{Z}$ to $M_{\mathrm{GUT}}$, clearly exhibiting mirage unification, is illustrated in Fig.15 $a$ ), for the MM-AMSB model with $n_{H}=1, n_{m}=1 / 2$ with $\alpha=6$, for which Eq. (1.6) gives $\mu_{\text {mir }} \sim 4 \times 10^{10} \mathrm{GeV}$. As discussed in Ref. [17, the unified value of gaugino masses at $\mu_{\text {mir }}$ is given by $M_{s}$, while the values of the first/second generation scalar mass parameters extrapolated to $\mu_{\text {mir }}$ are given by $m_{i}^{2}\left(\mu_{\text {mir }}\right)=\left(1-n_{i}\right) M_{s}^{2}$, so that the ratio of scalar-to-gaugino masses at $\mu_{\text {mir }}$ yields

$$
\left.\frac{m_{i}^{2}}{M_{a}^{2}}\right|_{\mu_{\mathrm{mir}}}=1-n_{i},
$$

which directly measures that matter field modular weights, and hence their location in the extra-dimensional geometry. We should mention though that even with ideal experimental measurements, we would not expect mirage unification to be perfect because,

1. mirage unification is a 1-loop phenomenon, and even if the RGEs are evolved at 1loop, the measured physical masses, and hence the extracted SSB parameters at the weak scale include "higher loop effects", and

2. gauge couplings do not unify exactly because of weak and GUT scale threshold corrections.

If $\alpha<0$ then $\mu_{\mathrm{mir}}>M_{\mathrm{GUT}}$ and gaugino and scalar mass parameters would have to be evolved beyond $M_{\mathrm{GUT}}$ using RGEs corresponding to $S U(3) \times S U(2) \times U(1)$ MSSM evolution to reveal mirage unification. For large negative values of $\alpha$, this is again straightforward, as illustrated in Fig. 15 b) for Point 3 in Table 1. We see that the gaugino mass parameters exhibit mirage unification beyond $Q=M_{\mathrm{GUT}}$ at $\mu_{\mathrm{mir}} \sim 10^{20} \mathrm{GeV}$. Matter scalar mass parameters can be similarly evolved to $\mu_{\text {mir }}$ in order to extract matter modular weights, within errors expected from the extrapolation.

However, if $\alpha$ is small in magnitude, but negative, we face a new problem because $\mu_{\text {mir }}$ becomes so large that one (or more) of the one-loop-evolved gauge couplings diverges for $Q<\mu_{\text {mir }}$, making the numerical integration of the RGEs from the weak scale to $\mu_{\text {mir }}$ difficult. We can circumvent this difficulty because it is possible to analytically integrate the one loop RGEs for gaugino masses and gauge couplings to obtain [64]

$$
\frac{M_{i}(Q)}{g_{i}^{2}(Q)}=K_{i}, \quad \text { or equivalently, } \frac{1}{M_{i}(Q)}=K_{i}^{-1} \times \frac{1}{g_{i}^{2}(Q)}
$$

where $K_{i}$ is a constant independent of the energy scale $Q$, and $i$ labels the gauge group. Indeed, if gaugino masses and gauge couplings both unify at $M_{\mathrm{GUT}}$, this leads to the well-known gaugino mass unification relation. From the experimentally determined weak scale values of $M_{i}$ and $g_{i}$ we can obtain $K_{i}$; these can then be used together with $1 / g_{i}^{2}(Q)$ obtained using

$$
\frac{1}{g_{i}^{2}(Q)}-\frac{1}{g_{i}^{2}\left(Q_{0}\right)}=-\frac{b_{i}}{8 \pi^{2}} \ln \left(\frac{Q}{Q_{0}}\right)
$$


to get the values of $1 / M_{i}(Q)$ at any higher scale. ${ }^{5}$ In Fig.15 $\left.c\right)$ we show the evolution of $1 / M_{i}(Q)^{2}$ using the procedure just described for a WMAP/LEP2-allowed case with $\left(n_{H}, n_{m}\right)=(0,0)$ with $\alpha=-1.635, m_{3 / 2}=25 \mathrm{TeV}, \tan \beta=10$ and $\mu>0$ (Point 4 from Table 1 of Ref. [何), which should have a value $\mu_{\text {mir }} \sim 2 \times 10^{37} \mathrm{GeV}$, to be compared with $Q \sim 10^{39} \mathrm{GeV}$ in the figure. Notice also that by working with $M_{i}(Q)^{-1}$ and $1 / g_{i}^{2}(Q)$, we circumvent the divergence that would have been present at $Q \sim 10^{27} \mathrm{GeV}$. The bands correspond to a hypothetical uncertainty of $\pm 2 \%$ on the extraction of the weak scale values of $M_{1}$ and $M_{2}$, and of $\pm 5 \%$ on the extraction of $M_{3}$.

Turning to the possibility of checking mirage unification for first/second generation scalar masses (for which Yukawa interactions can be neglected) when $\alpha<0$, we observe that

$$
m_{i}^{2}(Q)=Z_{i}-2 \sum_{a} \frac{C_{2}^{a}\left(f_{i}\right)}{b_{a}} M_{a}^{2}(Q)
$$

is a solution to the 1-loop RGEs for scalar mass squared, where $Z_{i}$ are scale-independent constants, and $C_{2}^{a}\left(f_{i}\right)$ is the quadratic Casimir for the $\mathrm{a}^{\text {th }}$ gauge group that we have encountered below Eq. (1.4). Since $Z_{i}$ are scale-independent, by taking the difference of $m_{i}^{2}(\mu)$ at the two scales, we obtain,

$$
m_{i}^{2}\left(\mu_{\text {mir }}\right)=m_{i}^{2}\left(\mu_{\text {weak }}\right)+2 \sum_{a} \frac{C_{2}^{a}\left(f_{i}\right)}{b_{a}}\left[M_{a}^{2}\left(\mu_{\text {weak }}\right)-M_{a}^{2}\left(\mu_{\text {mir }}\right)\right],
$$

where $m_{i}^{2}\left(\mu_{\text {weak }}\right)$ is the weak scale SSB parameter for the $i^{t h}$ sfermion, which we can obtain from its measured mass by removing the $D$-term. ${ }^{6}$ Eq. (6.4), or equivalently, Eq. (6.5) can then be used to obtain $m_{i}^{2}\left(\mu_{\text {mir }}\right)$ using the values of gaugino mass parameters at the weak and mirage unification scales that we have already obtained, and the corresponding modular weights can be extracted. Specifically, we first use the weak scale SSB mass parameters to obtain $Z_{i}$, and then use this value of $Z_{i}$ along with the gaugino SSB parameters at $Q=\mu_{\text {mir }}$ determined above, to obtain $m_{i}^{2}\left(\mu_{\text {mir }}\right)$.

We have performed a case study to illustrate this procedure, adopting the point chosen in Fig 15 c), except that we perform the analysis for $n_{m}=0, \frac{1}{2}$ and 1 to study how well the matter modular weights can be extracted in this difficult case. Very optimistically, we assume that it will eventually be possible to obtain colored sparticle masses to $\pm 5 \%$, that selectron masses will be measured with a precision of $\pm 1 \%$, and finally, that we will be able to extract weak scale values of $M_{1}$ and $M_{2}$ to $\pm 2 \%$. From these sfermion masses we obtain the corresponding weak scale SSB parameters upon removing the $D$-terms using $\cos 2 \beta=-1$, and then use Eq. (6.4) to get the SSB parameters at the mirage unification scale, $\mu_{\text {mir }}$ obtained from the intersection of $M_{1}$ and $M_{2}$ (as e.g. in Fig. 15c). The results of

\footnotetext{
${ }^{5}$ Notice that $\frac{1}{g_{i}^{2}(Q)}$ vanishes (i.e. $g_{i}^{2}(Q)$ blows up) when $\frac{1}{g_{i}^{2}\left(Q_{0}\right)}-\frac{b_{i}}{8 \pi^{2}} \ln \left(\frac{Q}{Q_{0}}\right)=0$, and becomes negative for yet larger values of $Q$. It is these (unphysical) negative values of $g_{i}^{2}(Q)$, obtained from the solution of the one-loop RGEs, that must be used in evaluating $1 / M_{i}(Q)$. The ratio of two different gaugino mass parameters can flip sign precisely because one of the corresponding $g_{i}^{2}(Q)$ s becomes negative.

${ }^{6}$ In practice we may not know the value of $\tan \beta$ very well. Fortunately, the change in the $D$-term is negligibly small as long as $\tan \beta \gtrsim 5$, and often even for smaller values of $\tan \beta$. In our case study described below, we use the $D$-term with $\cos 2 \beta=-1$.
} 


\begin{tabular}{lccc}
\hline & $n_{m}=0$ & $n_{m}=\frac{1}{2}$ & $n_{m}=1$ \\
\hline$\tilde{Q}$ & $465_{-576}^{+203}$ & $429_{-631}^{+211}$ & $388_{-654}^{+222}$ \\
$n_{\tilde{Q}}^{\exp }$ & {$[-7.5,1.2]$} & {$[-6.9,1.7]$} & {$[-6.2,2.4]$} \\
\hline$\tilde{e}_{L}$ & $280.1_{-8.9}^{+8.6}$ & $212.8_{-10.0}^{+9.6}$ & $110.4_{-17.1}^{+14.6}$ \\
$n_{\tilde{e}_{L}}^{\exp }$ & {$[-0.58,-0.29]$} & {$[0.05,0.27]$} & {$[0.70,0.84]$} \\
\hline$\tilde{e}_{R}$ & $257.6_{-3.5}^{+3.4}$ & $181.5_{-3.1}^{+3.0}$ & $-32.3_{-6.1}^{+7.6}$ \\
$n_{\tilde{e}_{R}}^{\exp }$ & {$[-0.26,-0.13]$} & {$[0.34,0.43]$} & {$[1.01,1.03]$} \\
\hline
\end{tabular}

Table 2: Values of sfermion mass parameters at $Q=\mu_{\text {mir }}$ obtained from the intersection of $M_{1}\left(\mu_{\text {mir }}\right)=M_{2}\left(\mu_{\text {mir }}\right)$ for the case studies with $\alpha=-1.635, m_{3 / 2}=25 \mathrm{TeV}, \tan \beta=10, \mu>0$, $n_{H}=0$, and $n_{m}=0, \frac{1}{2}$, and 1 . For each sparticle, we show the value of $\operatorname{sign}\left[m_{i}^{2}\left(\mu_{\mathrm{mir}}\right)\right] \sqrt{\left|m_{i}^{2}\left(\mu_{\mathrm{mir}}\right)\right|}$. The range corresponds to the projected experimental uncertainties discussed in the text. For each sparticle, the second line gives the range of the modular weight consistent with the range of the corresponding $m_{i}^{2}\left(\mu_{\text {mir }}\right)$. The negative sign in the value of $m\left(\tilde{e}_{R}\right)$ for $n_{m}=1$ really refers to the negative sign of $m^{2}\left(\tilde{e}_{R}\right)$.

our computation are shown in Table 2. The quoted range on the entries corresponds to the variation we obtain by varying the weak scale SSB parameters within their assumed errors. For each sparticle, on the second line, we show the range of modular weights compatible with the extracted values of the SSB mass squared parameters at the mirage unification scale. The following seem to be worthy of note.

- Even with the optimistic error of only $\pm 5 \%$ on the extraction of colored sparticle masses, we see that squark SSB parameters are very poorly restricted at the mirage scale. The reason is clear from Eq. (6.5): squarks and gluinos each have masses $\sim 1000 \mathrm{GeV}$, so that a very large cancellation between the terms on the right hand side is needed to get the left hand side to be $\sim(100-250 \mathrm{GeV})^{2}$, the value of the matter mass parameters at $\mu_{\mathrm{mir}}$. The effect of higher loop terms, not-exact-unification of gauge couplings and various threshold corrections spoil the delicate cancellations, leading to a very large error on the extraction of squark modular weights in this case.

- We see that while slepton masses do appear to unify within $\lesssim 10 \%$ at least for $n_{m}=0$ and $n_{m}=1 / 2$ cases in Table 2, the ranges of the modular weights that we extract using our procedure are shifted systematically to lower values. We have traced this shift to the fact that the value of $M_{a}\left(\mu_{\text {mir }}\right) \simeq 232.5 \mathrm{GeV}$ that we obtain from Fig. 15 c) is too small by about $10 \%$, causing a systematic shift in the extracted modular weights. We had already remarked that $\mu_{\text {mir }}$ obtained from this figure was about 50 times larger than its theoretically expected value. For $n_{m}=1$, it appears that $m^{2}\left(\tilde{e}_{R}\right)$ and $m^{2}\left(\tilde{e}_{L}\right)$ unify very poorly. Note, however, that $m_{i}^{2}\left(\mu_{\text {mir }}\right)$ is expected to be very small in this case, so that we must again have a large cancellation between the two terms on the right-hand-side of Eq. (6.5) for this to occur. As with the squarks, systematic effects then play an important part. It is, however, interesting to see that $m_{i}^{2}\left(\mu_{\text {mir }}\right) \ll M\left(\mu_{\text {mir }}\right)$, so that the extracted matter modular weights are close to unity. 
We conclude that while our simple procedure provides a good indication of mirage unification of gaugino mass parameters, and a qualitative indication for the mirage unification of scalar mass parameters, quantitative extraction of the model parameters have significant systematic uncertainties in this difficult case of very large $\mu_{\mathrm{mir}}$. We presume that this is because of higher loop and finite correction effects that are present in the weak scale SSB parameters. In principle, a determination of all weak scale SSB parameters may make it possible to extract "one-loop" weak scale gaugino and first/second generation SSB parameters, but until this is possible, quantitative extraction of modular weights (particularly with a precision to enable a distinction between toroidal and more complicated compactifications) may prove difficult if $\mu_{\mathrm{mir}} \gg M_{\mathrm{GUT}}$.

\section{Summary and Conclusions}

Mixed modulus-anomaly mediation of SUSY breaking, also referred to as mirage-mediation, provides a new, theoretically well-motivated, and phenomenologically viable framework for analyses of the implications of SUSY. The MM-AMSB model is completely specified by just three parameters along with the discrete set of modular weights for chiral super-fields that are determined by their location in the extra spatial dimensions. The naturally heavy moduli and gravitinos can be consistent with cosmology, assuming that there is a second late period of inflation that dilutes their density. The observed dark matter may then comprise principally of neutralinos that are thermally produced upon reheating after this epoch. The framework also provides a plausible solution to the SUSY flavor problem, and also ameliorates the SUSY CP problem.

We have examined the phenomenology of these models under the assumption (motivated by the absence of large flavor changing neutral currents) that matter superfields all reside on either a $D 7$ brane, a $D 3$ brane, or on their intersection: using examples with toroidal compactifications, the matter modular weights are fixed to be 0,1 or $\frac{1}{2}$, respectively. Higgs superfields may lie at a different location, giving us 9 discrete choices for set $\left(n_{H}, n_{m}\right)$ of the matter and Higgs modular weights. More complicated configurations that lead to other choices for the modular weights (e.g. 2/3) may be possible, but we expect that the phenomenology will then "lie in between" the cases that we examine.

The phenomenological implications of the model differ from that of other models because, (1) the combination of modulus and anomaly-mediated contributions to SUSY breaking lead to novel patterns of sparticle masses, and (2) the composition of the lightest neutralino differs because the weak scale gaugino masses are not in the ratio $\sim 1: 2: 6$ predicted by the gaugino mass unification condition, as is the case in many models. These features of the spectrum make it possible to obtain a neutralino thermal relic density consistent with WMAP measurements in a variety of interesting ways that have been proposed in previous bottom-up studies, not all of which are possible in the much studied mSUGRA model. These include co-annihilation with staus or stops, resonance annihilation via $A$ and $H$, bino-wino co-annihilation, mixed higgsino and mixed bino-wino-higgsino DM and low $\left|M_{3}\right|$ (mixed-higgsino) DM, but not all mechanisms are realized for every choice of matter and Higgs modular weights. Interestingly, mixed wino DM (without also a significant hig- 
gsino content), or of mixed higgsino DM via non-universal Higgs mass parameters, does not seem to be realized within this framework.

Within this framework, it may be possible for Tevatron experiments to discover supersymmetry in portions of the parameter space not accessible to LEP2 searches. Possibilities include searches via the $E_{T}^{\text {miss }}$ channel for say $\left(n_{H}, n_{m}\right)=(1,0)$, where gluinos and squarks as low as about $300 \mathrm{GeV}$ may have escaped detection up to now, searches for light stops and, depending the branching ratio for $\widetilde{Z}_{2} \rightarrow \ell \ell \widetilde{Z}_{1}$ decay, trilepton events from $\widetilde{W}_{1} \widetilde{Z}_{2}$ production. As can be seen from Fig. ․ㅏFig. 3, assuming $100 \mathrm{fb}^{-1}$ of integrated luminosity, LHC experiments should be able to detect SUSY signals over most of the parameter space of the model consistent the relic density measurement, with some exceptions for $n_{H}=0$ or $1 / 2$, and $n_{m}=1$. Experiments at a $1 \mathrm{TeV}$ linear collider will be able to make precision measurements over part of the region probed by the LHC. Interestingly, these experiments will be able to explore charginos and neutralinos if the measured relic density arises due to BWCA: this is important because the small gap, $m_{\widetilde{W}_{1}}-m_{\widetilde{Z}_{1}}$, may make exploration of charginos difficult at the LHC.

We have also examined prospects for direct and indirect detection of DM for the nine cases of modular weights. Our results for direct detection are shown in Fig. 11, where we see that the CDMS experiment has already begun to exclude a portion of the parameter space for $m_{\widetilde{Z}_{1}}$ as heavy as $300 \mathrm{GeV}$, for the larger value of $\tan \beta$. It is also striking that proposed ton size noble gas detectors (we use the proposed Warm Argon Project with $1,400 \mathrm{~kg}$ of argon as a benchmark) will be able to explore most of the parameter space of this model, even if $m_{\widetilde{Z}_{1}}$ is as heavy as $\sim 1000 \mathrm{GeV}$. Prospects for indirect detection are somewhat less certain. Models with significant higgsino components yield the largest signals, while models with a bino LSP will generally not give an observable signal. There may be detectable signals from high energy muons from the sun at IceCube, as well as from anti-particles at Pamela and Gaps, or from gamma rays in GLAST. There is, however, considerable theoretical uncertainty (factor 10-20) in the prediction of the anti-particle signals from the unknown clumping of neutralinos in our galactic halo, and up to four orders of magnitude uncertainty for signals at GLAST, so that projections for these experiments should be viewed with care. Since this clumping provides a common uncertainty for these experiments, observing a signal in one of these will greatly facilitate a much more certain prediction for the other experiments, assuming that the observed signal is from neutralinos annihilating in our galactic halo.

Finally, we have explored the possibility of testing mirage unification of SSB parameters, which is the smoking gun for this framework. The case for small or intermediate mirage unification scale had already been explored and it had been shown that mirage unification of both gaugino and scalar mass parameters would be readily testable, and allow extraction of the modular weights. Here, we have explored the much more difficult case that occurs if $\alpha$ is small in magnitude, but negative, so that $\mu_{\mathrm{mir}} \gg M_{\mathrm{GUT}}$. In this case, we find that mirage unification of gaugino masses should still be testable, although extraction of underlying values of $\mu_{\text {mir }}$ and $M_{s}$ has significant systematic uncertainty due to the large "distance" between weak and mirage scales. Combining measurements from the LHC with measurements that will be possible with initial runs of a linear collider may allow a quali- 
tative test of mirage unification of scalar masses, but unlike the case of intermediate values of $\mu_{\mathrm{mir}}$, extraction of matter modular weights is fraught with systematic uncertainty.

In summary, we have made an exhaustive exploration of the phenomenology of the MM-AMSB model, a promising new framework for SUSY phenomenology. The novel patterns of sparticle masses that are possible allow various mechanisms that lead to a DM relic density in agreement with observations to be incorporated into a top-down framework, something not previously possible. This can qualitatively change the correlations between various experimental signals from expectations in the much studied mSUGRA framework. In view of its attractive and economic theoretical structure as well as its attractive phenomenological attributes, we believe it is at least as attractive (and theoretically, perhaps, more compelling) as mSUGRA as a framework for analyses of SUSY.

\section{Acknowledgments}

We thank Kiwoon Choi for clarifying discussions and correspondence. We are also grateful to J. Conlon, M. Endo and F. Takahashi for drawing our attention to their work, and for helpful correspondence about the same. This research was supported in part by the U.S. Department of Energy grants DE-FG02-97ER41022 and DE-FG02-04ER41291.

\section{References}

[1] S. Kachru, R. Kallosh, A. Linde and S. P. Trivedi, Phys. Rev. D 68 (2003) 046005.

[2] K. Choi, A. Falkowski, H. P. Nilles, M. Olechowski and S. Pokorski, J. High Energy Phys. 0411 (2004) 076; K. Choi, A. Falkowski, H. P. Nilles and M. Olechowski, Nucl. Phys. B 718 (2005) 113.

[3] L. Randall and R. Sundrum, Nucl. Phys. B 557 (1999) 79; G. F. Giudice, M. Luty, H. Murayama and R. Rattazzi, J. High Energy Phys. 9812 (1998) 027.

[4] M. Endo, K. Hamaguchi and F. Takahashi, Phys. Rev. Lett. 96 (2006) 211201; S. Nakamura and M. Yamaguchi, Phys. Lett. B 638 (2006) 389; M. Dine, R. Kitano, A. Morisse and Y. Shirman, Phys. Rev. D 73 (2006) 123518.

[5] D. H. Lyth and E. D. Stewart, Phys. Rev. Lett. 75 (1995) 201 and Phys. Rev. D 53 (1996) 1784 .

[6] K. Choi, K-S. Jeong and K. Okumura, J. High Energy Phys. 0509 (2005) 039.

[7] P.G. Camara, L. E. Ibañez, A.M. Uranga, Nucl. Phys. B 689 (2004) 195 and Nucl. Phys. B 708 (2005) 268; L. E. Ibañez,Phys. Rev. D 71 (2005) 055005.

[8] J. P. Conlon, D. Cremades and F. Quevedo J. High Energy Phys. 0701 (2007) 022; J. P. Conlon, S. S. Abdussalam, F. Quevedo and K. Suruliz, J. High Energy Phys. 0701 (2007) 032 .

[9] K. Choi, K-S. Jeong, J. High Energy Phys. 0701 (2007) 103. K. Choi, K. S. Jeong, T. Kobayashi and K. i. Okumura, arXiv:hep-ph/0612258.

[10] ISAJET v7.74, by H. Baer, F. Paige, S. Protopopescu and X. Tata, hep-ph/0312045. 
[11] A. Falkowski, O. Lebedev and Y. Mambrini, J. High Energy Phys. 0511 (2005) 034; see hep-ph/0507110 v2 for a discussion of the possibility of the light stop.

[12] M. Endo, M. Yamaguchi and K. Yoshioka, Phys. Rev. D 72 (2005) 015004.

[13] H. Baer, E. Park, X. Tata and T. T. Wang, J. High Energy Phys. 0608 (2006) 041.

[14] K. Choi, K. S. Jeong, T. Kobayashi and K. i. Okumura, Phys. Lett. B 633 (2006) 355; R. Kitano and Y. Nomura, Phys. Lett. B 631 (2005) 58, and Phys. Rev. D 73 (2006) 095004.

[15] D. N. Spergel et al., astro-ph/0603449 (2006); see D. N. Spergel et al., astro-ph/0302209 (2003) for results from the analysis of the first year of WMAP data.

[16] K. Kawagoe and M. Nojiri, Phys. Rev. D 74 (2006) 115011.

[17] H. Baer, E. Park, X. Tata and T. T. Wang, Phys. Lett. B 641 (2006) 447.

[18] K. Choi, K. Y. Lee, Y. Shimizu, Y. G. Kim and K. Okumura, JCAP0612 (2006) $017 .$.

[19] H. Baer, J. Ferrandis, S. Kraml and W. Porod, Phys. Rev. D 73 (2006) 015010.

[20] H. Baer, C. Balazs and A. Belyaev, J. High Energy Phys. 0202 (2002) 042.

[21] P. Gondolo, J. Edsjo, P. Ullio, L. Bergstrom, M. Schelke and E. A. Baltz, JCAP 0407 (2004) 008 [arXiv:astro-ph/0406204].

[22] See e.g. Joint LEP SUSY Working Group, http://lepsusy.web.cern.ch/lepsusy/

[23] A. Belyaev, Q-H. Cao, D. Nomura, K. Tobe and C.-P. Yuan, hep-ph/0609079 (2006).

[24] H. Baer, T. Krupovnickas, A. Mustafayev, E. Park, S. Profumo and X. Tata, J. High Energy Phys. 0512 (2005) 011.

[25] H. Baer, C. H. Chen, F. Paige and X. Tata, Phys. Rev. D 52 (1995) 2746 and Phys. Rev. D 53 (1996) 6241; H. Baer, C. H. Chen, M. Drees, F. Paige and X. Tata, Phys. Rev. D 59 (1999) 055014; S. Abdullin and F. Charles, Nucl. Phys. B 547 (1999) 60; S. Abdullin et al. (CMS Collaboration), hep-ph/9806366; B. Allanach, J. Hetherington, A. Parker and B. Webber, J. High Energy Phys. 08 (2000) 017; for a recent update, see H. Baer, C. Balazs, A. Belyaev, T. Krupovnickas and X. Tata, J. High Energy Phys. 0306 (2003) 054. See also P. G. Mercadante, J. K. Mizukoshi and X. Tata, Phys. Rev. D 72 (2005) 035009.

[26] H. Baer, A. Belyaev, T. Krupovnickas and X. Tata, J. High Energy Phys. 0402 (2004) 007, H. Baer, T. Krupovnickas and X. Tata, J. High Energy Phys. 0406 (2004) 061.

[27] M. Drees and M. Nojiri, Phys. Rev. D 47 (1993) 376; H. Baer and M. Brhlik, Phys. Rev. D 57 (1998) 567; H. Baer, M. Brhlik, M. Diaz, J. Ferrandis, P. Mercadante, P. Quintana and X. Tata, Phys. Rev. D 63 (2001) 015007; J. Ellis, T. Falk, G. Ganis, K. Olive and M. Srednicki, Phys. Lett. B 510 (2001) 236; L. Roszkowski, R. Ruiz de Austri and T. Nihei, J. High Energy Phys. 0108 (024) 2001; A. Djouadi, M. Drees and J. L. Kneur, J. High Energy Phys. 0108 (2001) 055; A. Lahanas and V. Spanos, Eur. Phys. J. C 23 (2002) 185.

[28] H. Baer, A. Mustafayev, E. Park, S. Profumo and X. Tata, J. High Energy Phys. 0604 (2006) 041.

[29] H. Baer, A. Mustafayev, S. Profumo and X. Tata, Phys. Rev. D 75 (2007) 035004.

[30] H. Baer, A. Mustafayev, E. Park and S. Profumo, J. High Energy Phys. 0507 (2005) 046.

[31] H. Baer, A. Mustafayev, S. Profumo, A. Belyaev and X. Tata, Phys. Rev. D 71 (2005) 095008 and J. High Energy Phys. 0507 (2005) 065. 
[32] G. W. Bennett et al. Phys. Rev. D 73 (2006) 072003.

[33] W. M. Yao et al. (Particle Data Group), J. Phys. G 33 (2006) 1.

[34] See e.g. H. Baer, C. Balazs, J. Ferrandis and X. Tata, Phys. Rev. D 64 (2001) 035004 based on T. Moroi, Phys. Rev. D 53 (1996) 6565 and Erratum-ibid. D56 (1997) 4424.

[35] See e.g. H. Baer and M. Brhlik, Phys. Rev. D 55 (1997) 3201 and H. Baer, M. Brhlik, D. Castano and X. Tata, Phys. Rev. D 58 (1998) 015007.

[36] E. Barberio et al. (Heavy Flavor Averaging Group), hep-ex/0603003.

[37] K. Chetyrkin, M. Misiak and M. Munz, Phys. Lett. B 400 (1997) 206 and erratum-ibid, Phys. Lett. B 425 (1998) 414; A. Buras et al., Phys. Lett. B 414 (1997) 157 and erratum-ibid, Phys. Lett. B 434 (1998) 459; A. Kagan and M. Neubert, Eur. Phys. J. C 7 (1999) 5.

[38] V. M. Abazov et al., Phys. Lett. B 638 (2006) 119.

[39] D. Dicus, S. Nandi and X. Tata, Phys. Lett. B 129 (1983) 451; A. Chamseddine, P. Nath and R. Arnowitt, Phys. Lett. B 129 (1983) 445; H. Baer and X. Tata,Phys. Lett. B 155 (1985) 278; H. Baer, K. Hagiwara and X. Tata, Phys. Rev. Lett. 57 (1986) 294 and Phys. Rev. D 35 (1987) 1598; R. Arnowitt and P. Nath, Mod. Phys. Lett. A 2 (1987) 331: R. Barbieri, F. Caravaglios, M. Frigeni and M. Mangano, Nucl. Phys. B 367 (1991) 28; H. Baer and X. Tata, Phys. Rev. D 47 (1993) 2739; J. Lopez, D. Nanopoulos, X. Wang and A. Zichichi, Phys. Rev. D 48 (1993) 2062 and Phys. Rev. D 52 (1995) 142; H. Baer, C. Kao and X. Tata, Phys. Rev. D 48 (1993) 5175; S. Mrenna, G. Kane, G. Kribs and J. Wells, Phys. Rev. D 53 (1996) 1168; H. Baer, C. H. Chen, F. Paige and X. Tata, Phys. Rev. D 54 (1996) 5866; K. Matchev and D. Pierce, Phys. Rev. D 60 (1999) 075004; H. Baer, M. Drees, F. Paige, P. Quintana and X. Tata, Phys. Rev. D 61 (2000) 095007; V. Barger, C. Kao and T. Li, Phys. Lett. B 433 (1998) 328; V. Barger and C. Kao, Phys. Rev. D 60 (1999) 115015; K. Matchev and D. Pierce, Phys. Lett. B 467 (1999) 225; H. Baer, T. Krupovnickas and X. Tata, J. High Energy Phys. 0307 (2003) 020.

[40] See J. Lopez et al., Ref. [39].

[41] H. Baer, J. Sender and X. Tata, hep-ph/9308376 and Phys. Rev. D 50 (1994) 4517; R. Demina, J. Lykken, K. Matchev and A. Nomerotski, Phys. Rev. D 62 (2000) 035011.

[42] M. Carena, M. Quiros, A. Riotto, I. Vilja and C. E. M. Wagner, Nucl. Phys. B 503 (1997) 387; M. Carena, M. Quiros and C. E. M. Wagner, Nucl. Phys. B 524 (1998) 3; M. Carena, M. Quiros, M. Seco and C. Wagner, Nucl. Phys. B 650 (2003) 24; C. Balazs, M. Carena and C. Wagner, Phys. Rev. D 70 (2004) 015007; for a review, see M. Trodden, hep-ph/0411301 (2004).

[43] H. Baer, K. Hagiwara and X. Tata, Phys. Rev. D 35 (1987) 1598; H. Baer, D. Dzialo-Karatas and X. Tata, Phys. Rev. D 42 (1990) 2259; H. Baer, C. Kao and X. Tata, Phys. Rev. D 48 (1993) 5175; H. Baer, C. H. Chen, F. Paige and X. Tata, Phys. Rev. D 50 (1994) 4508. I. Hinchliffe et al., Phys. Rev. D 55 (1997) 5520 and Phys. Rev. D 60 (1999) 095002; H. Bachacou, I. Hinchliffe and F. Paige, Phys. Rev. D 62 (2000) 015009; Atlas Collaboration, LHCC 99-14/15; C. Lester, M. Parker and M. White, J. High Energy Phys. 0601 (2006) 080.

[44] The Isatools neutralino-nucleon scattering rate is discussed in H. Baer, C. Balazs, A. Belyaev and J. O'Farrill, JCAP 0309 (2003) 007.

[45] D. S. Akerib et al. (CDMS Collaboration), astro-ph/0405033 (2004). 
[46] R. W. Schnee et al. (SuperCDMS Collaboration), astro-ph/0502435.

[47] See talk by C. Rubbia at 6th UCLA Symposium on Sources and Detection of Dark Matter and Dark Energy in the Universe, Marina del Ray, CA, February (2004).

[48] H. Baer and J. O'Farrill, JCAP0404 (2004) 005; H. Baer, A. Belyaev, T. Krupovnickas and J. O'Farrill, JCAP0408 (2004) 005.

[49] J. Ahrens et al., (IceCube Collaboration), Nucl. Phys. 118 (Proc. Suppl.) (2003) 388; F. Halzen, astro-ph/0311004; F. Halzen and D. Hooper, JCAP0401 (2004) 002.

[50] E. Carmona et al., (Antares Collaboration), Nucl. Phys. 95 (Proc. Suppl.) (2001) 161.

[51] J. Silk, K. Olive and M. Srednicki, Phys. Rev. Lett. 55 (1985) 257; K. Freese, Phys. Lett. B 167 (1986) 295; L. Krauss, M. Srednicki and F. Wilczek, Phys. Rev. D 33 (1986) 2079; V. Berezinsky, A. Bottino, J. R. Ellis, N. Fornengo, G. Mignola and S. Scopel, Astropart, Phys. 5 (1996) 333; L. Bergstrom, J. Edsjo and P. Gondolo, Phys. Rev. D 55 (1997) 1765 and Phys. Rev. D 58 (1998) 103519; A. Bottino, F. Donato, N. Fornengo and S. Scopel, Astropart. Phys. 10 (1999) 203; A. Corsetti and P. Nath, Int. J. Mod. Phys. A 15 (2000) 905; V. Barger, F. Halzen, D. Hooper and C. Kao, Phys. Rev. D 65 (2002) 075022; V. Bertin, E. Nezri and J. Orloff, Eur. Phys. J. C 26 (2002) 111 and J. High Energy Phys. 0302 (2003) 046; see also Ref. 486.

[52] M. A. DuVernois et al. (HEAT Collaboration), Astrophys. J. 559 (2001) 296.

[53] M. Pearce (Pamela Collaboration), Nucl. Phys. 113 (Proc. Suppl.) (2002) 314.

[54] J. Casaus et al. (AMS Collaboration), Nucl. Phys. 114 (Proc. Suppl.) (2003) 259.

[55] S. Orito et al. (BESS Collaboration), Phys. Rev. Lett. 84 (2000) 1078.

[56] H. Fuke et al. (BESS Collaboration), astro-ph/0504361.

[57] K. Mori, C. J. Hailey, E. A. Baltz, W. W. Craig, M. Kamionkowski, W. T. Serber and P. Ullio, Astrophys. J. 566 (2002) 604, C. J. Hailey et al., JCAP 0601 (2006) 007; H. Baer and S. Profumo, JCAP0512 (2005) 008.

[58] S. Profumo and P. Ullio, JCAP 0407 (2004) 006; S. Profumo and C.E. Yaguna, Phys. Rev. D 70 (2004) 095004.

[59] A. El-Zant, I. Shlosman and Y. Hoffman, Astrophys. J. 560 (2001) 336; A. Burkert, Astrophys. J. 447 (1995) L25.

[60] J.F. Navarro et al., MNRAS, 349 (2004) 1039; B. Moore, et al.,Astrophys. J. Lett. 524, L19 (1999).

[61] H. A. Mayer-Hasselwander et al. (EGRET Collaboration), MPE-440 (1998).

[62] F. Stecker, Phys. Lett. B 201 (1988) 529; F. W. Stecker and A. J. Tylka, Astrophys. J. 343 (1989) 169; S. Rudaz and F. Stecker, Astrophys. J. 368 (1991) 406; M. Urban et al., Phys. Lett. B 293 (1992) 149; V. Berezinsky, A. Gurevich and K. Zybin, Phys. Lett. B 294 (1992) 221; V. Berezinsky, A. Bottino and G. Mignola, Phys. Lett. B 325 (1994) 136; L. Bergstrom, P. Ullio and J. H. Buckley, Astropart. Phys. 9 (1998) 137; L. Bergstrom, J. Edsjö and P. Ullio, Phys. Rev. D 58 (1998) 083507; J. Buckley et al., astro-ph/0201160; P. Ullio, L. Bergstrom, J. Edsjö and C. Lacey, Phys. Rev. D 66 (2002) 123502; see also Ref. 48].

[63] A. Morselli et al., (GLAST Collaboration), Nucl. Phys. 113 (Proc. Suppl.) (2002) 213.

[64] See e.g. H. Baer and X. Tata, Weak Scale Supersymmetry, Cambridge University Press (2006). 

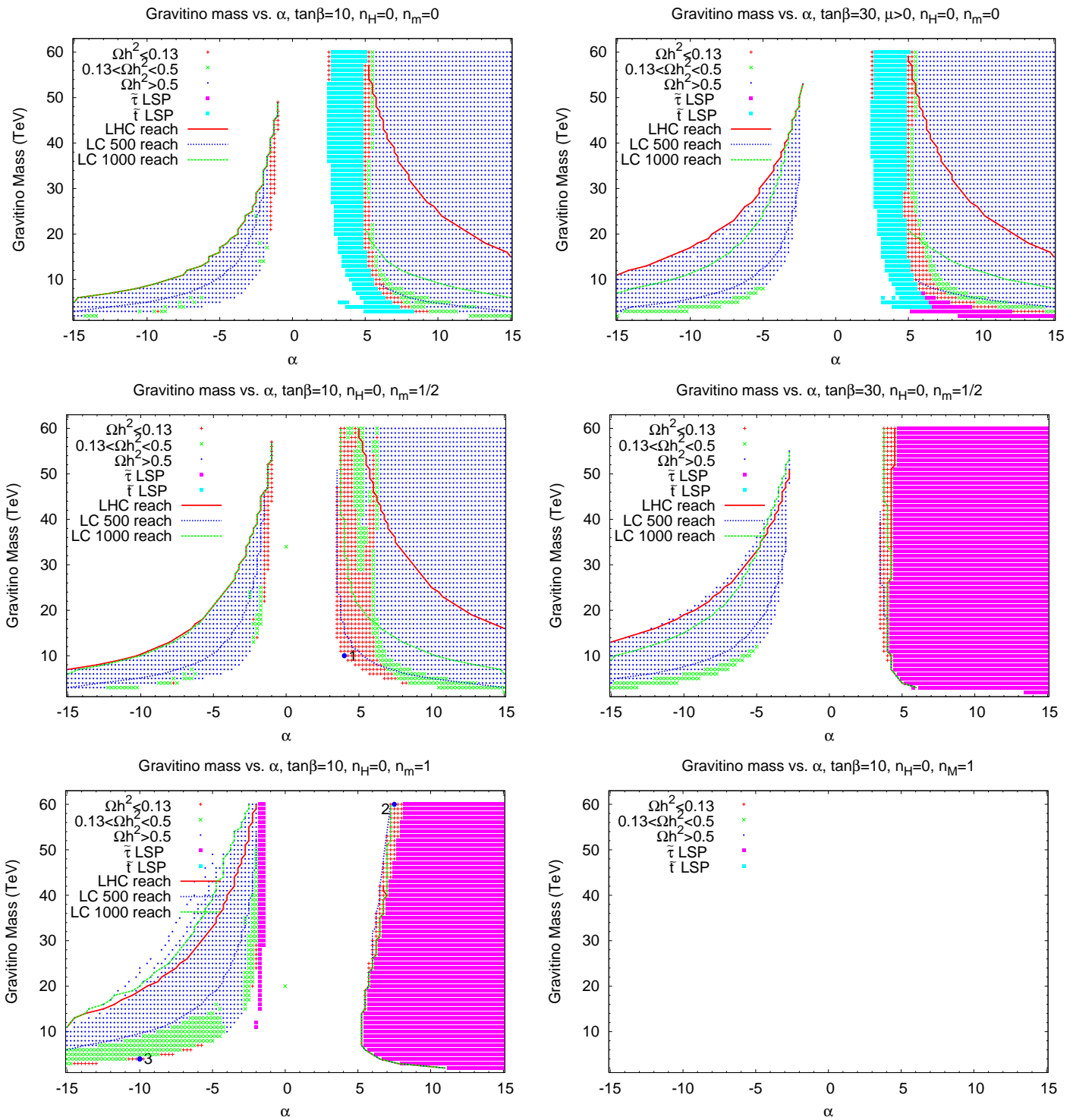

Figure 1: Allowed regions of parameter space of the MM-AMSB model with modular weight $n_{H}=0$ and $\left.\left.\left.\left.a\right), b\right) n_{m}=0, c\right), d\right) n_{m}=1 / 2$ and $e$ ), f) $n_{m}=1$. Frames $a$ ), c) and $e$ ) have $\tan \beta=10$ while $b), d$ ) and $f$ ) have $\tan \beta=30$. The plots also show the expected thermal neutralino relic density from the Big Bang, and the reach of the CERN LHC for $100 \mathrm{fb}^{-1}$ and for a $\sqrt{s}=500$ and $1000 \mathrm{GeV}$ linear $e^{+} e^{-}$collider. We take $\mu>0$ and $m_{t}=175 \mathrm{GeV}$. 

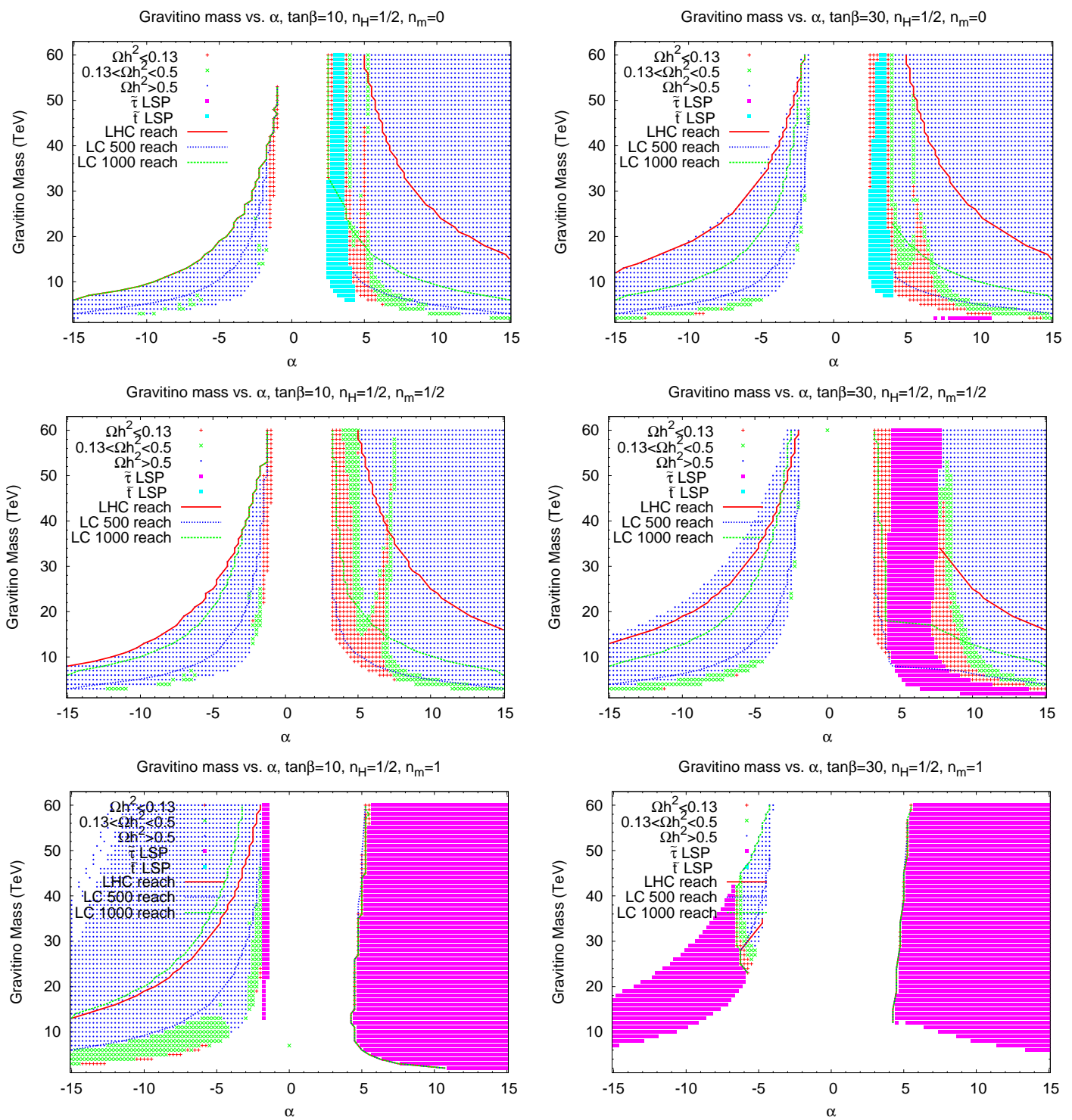

Figure 2: Allowed regions of parameter space of the MM-AMSB model with modular weight $n_{H}=1 / 2$ and $a$ ), b) $\left.\left.n_{m}=0, c\right), d\right) n_{m}=1 / 2$ and $e$ ), f) $n_{m}=1$. Frames $a$ ), c) and $e$ ) have $\tan \beta=10$ while $b$ ), d) and $f$ ) have $\tan \beta=30$. The plots also show the expected thermal neutralino relic density from the Big Bang, and the reach of the CERN LHC for $100 \mathrm{fb}^{-1}$ and for a $\sqrt{s}=500$ and $1000 \mathrm{GeV}$ linear $e^{+} e^{-}$collider. We take $\mu>0$ and $m_{t}=175 \mathrm{GeV}$. 

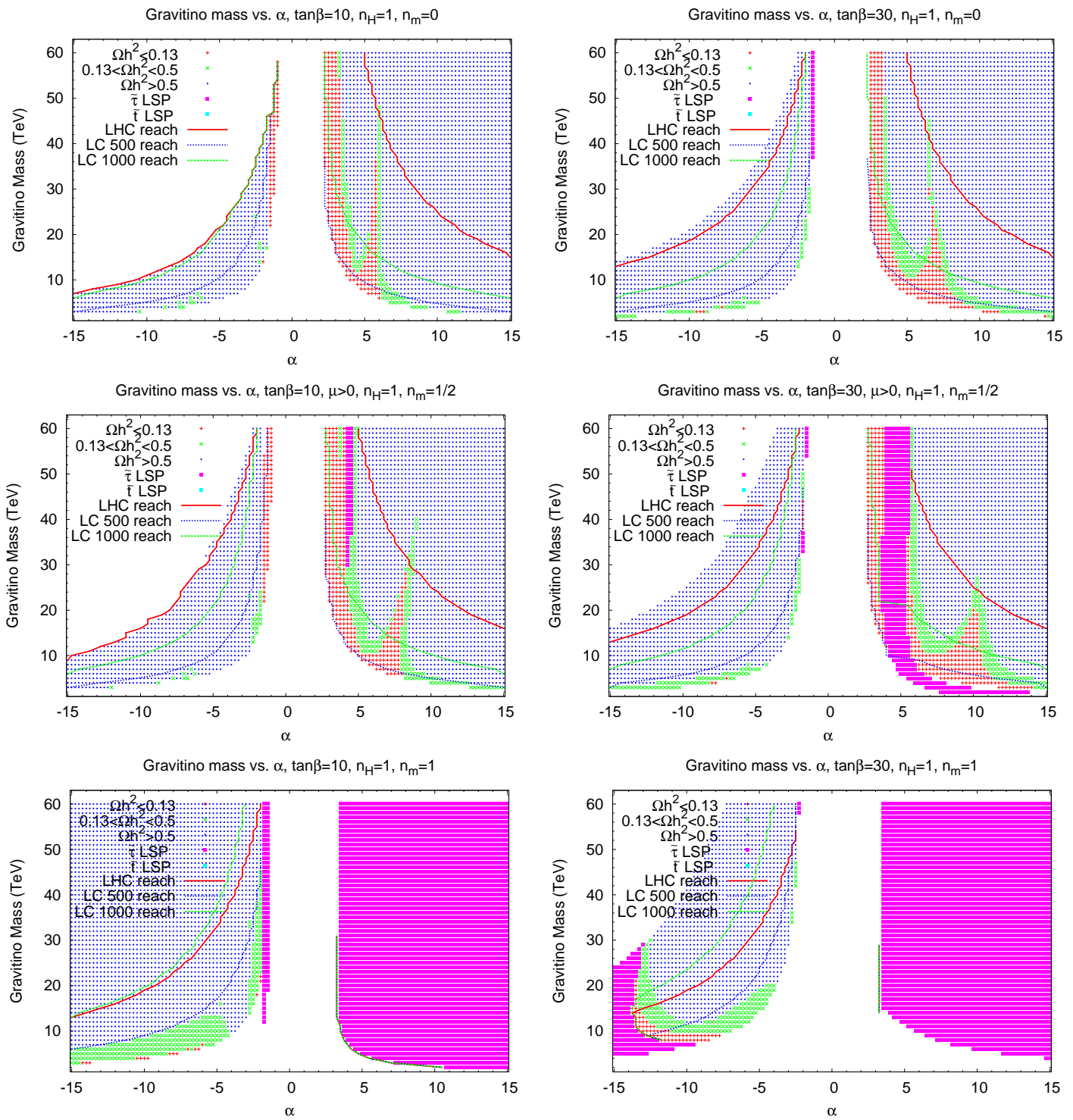

Figure 3: Allowed regions of parameter space of the MM-AMSB model with modular weight $n_{H}=1$ and $\left.\left.\left.\left.a\right), b\right) n_{m}=0, c\right), d\right) n_{m}=1 / 2$ and $e$ ), f) $n_{m}=1$. Frames $a$ ), c) and $e$ ) have $\tan \beta=10$ while $b), d$ ) and $f$ ) have $\tan \beta=30$. The plots also indicate the neutralino relic density, and the reach of the CERN LHC for $100 \mathrm{fb}^{-1}$ and a $\sqrt{s}=500$ and $1000 \mathrm{GeV}$ linear $e^{+} e^{-}$collider. We also take $\mu>0$ and $m_{t}=175 \mathrm{GeV}$. 

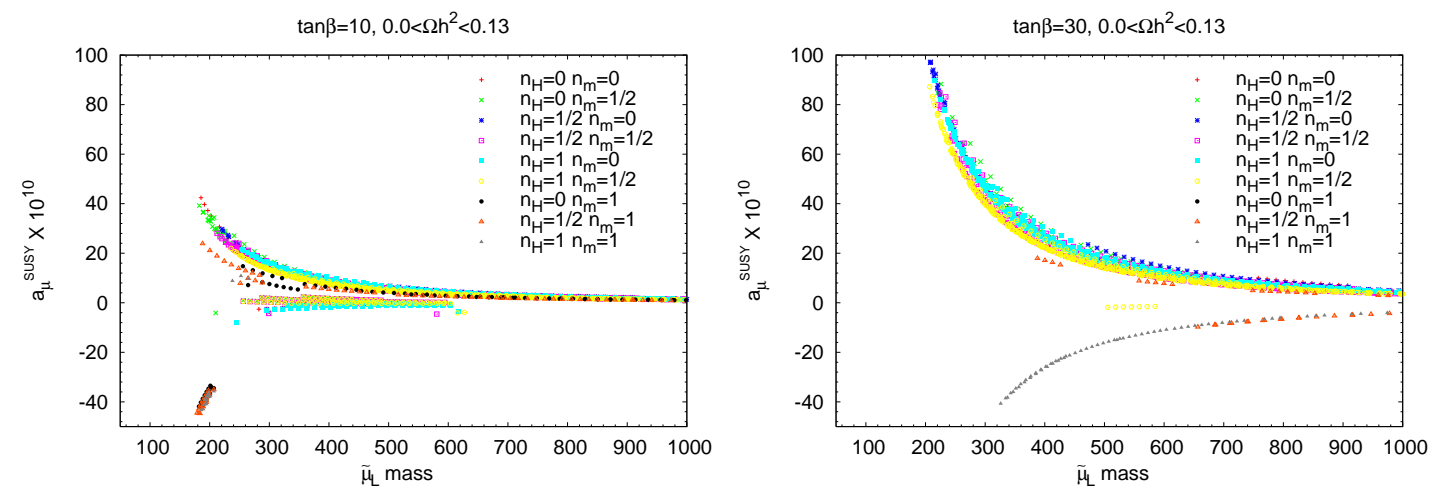

Figure 4: Plot of $a_{\mu}^{\mathrm{SUSY}}$ vs. $m_{\tilde{\mu}_{L}}$ in the MM-AMSB model for various modular weight choices with $\mu>0$ and $m_{t}=175 \mathrm{GeV}$. Only models with $\Omega_{\widetilde{Z}_{1}} h^{2}<0.13$ are retained. In frame $a$ ), we show results for $\tan \beta=10$, while in frame $b$ ) we take $\tan \beta=30$.
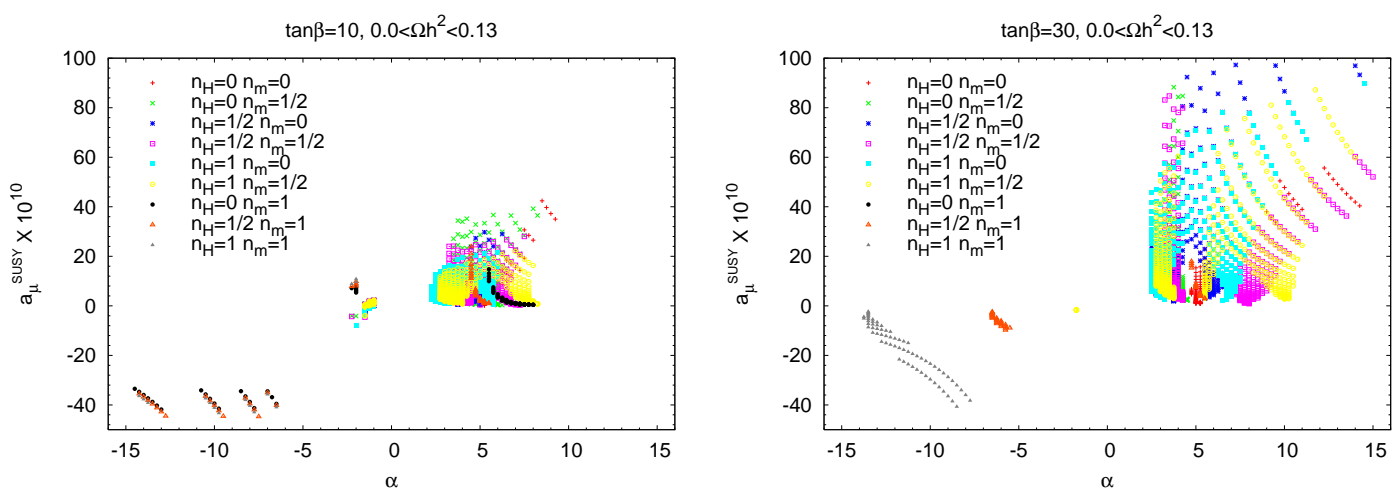

Figure 5: Plot of $a_{\mu}^{\text {SUSY }}$ vs. $\alpha$ in the MM-AMSB model for various modular weight choices with $\mu>0$ and $m_{t}=175 \mathrm{GeV}$. Only models with $\Omega_{\widetilde{Z}_{1}} h^{2}<0.13$ are retained. In frame $a$ ), we show results for $\tan \beta=10$, while in frame $b$ ) we take $\tan \beta=30$.
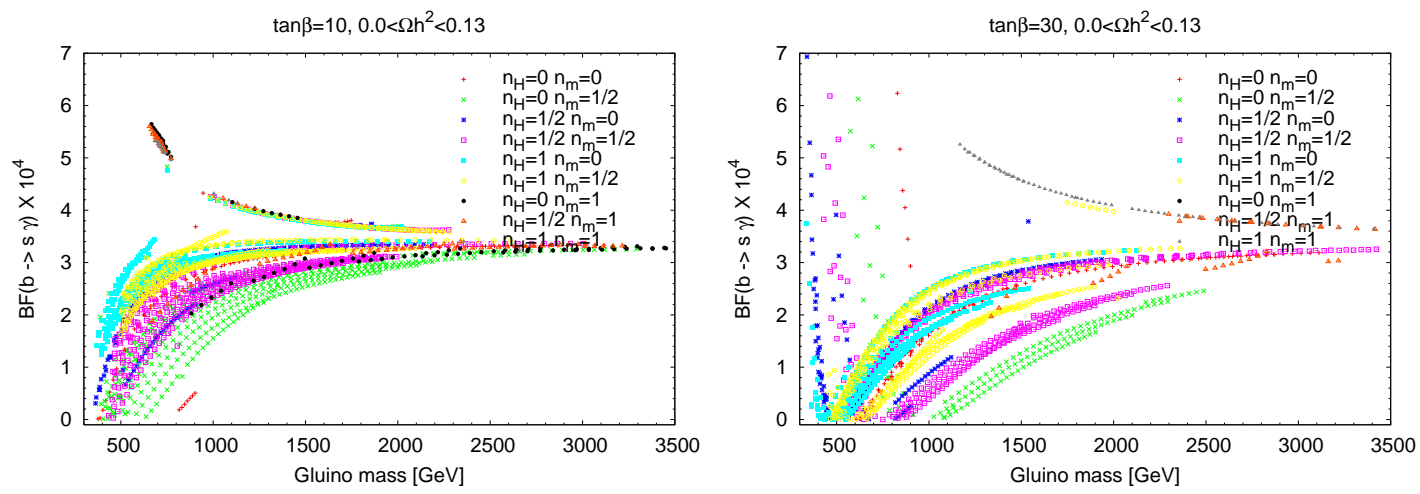

Figure 6: Plot of $B F(b \rightarrow s \gamma)$ vs. $m_{\tilde{g}}$ in the MM-AMSB model for the different modular weight choices, for $a) \tan \beta=10$, and $b) \tan \beta=30$. Only models with $\Omega_{\widetilde{Z}_{1}} h^{2}<0.13$ are included. We take $\mu>0$ and $m_{t}=175 \mathrm{GeV}$. 

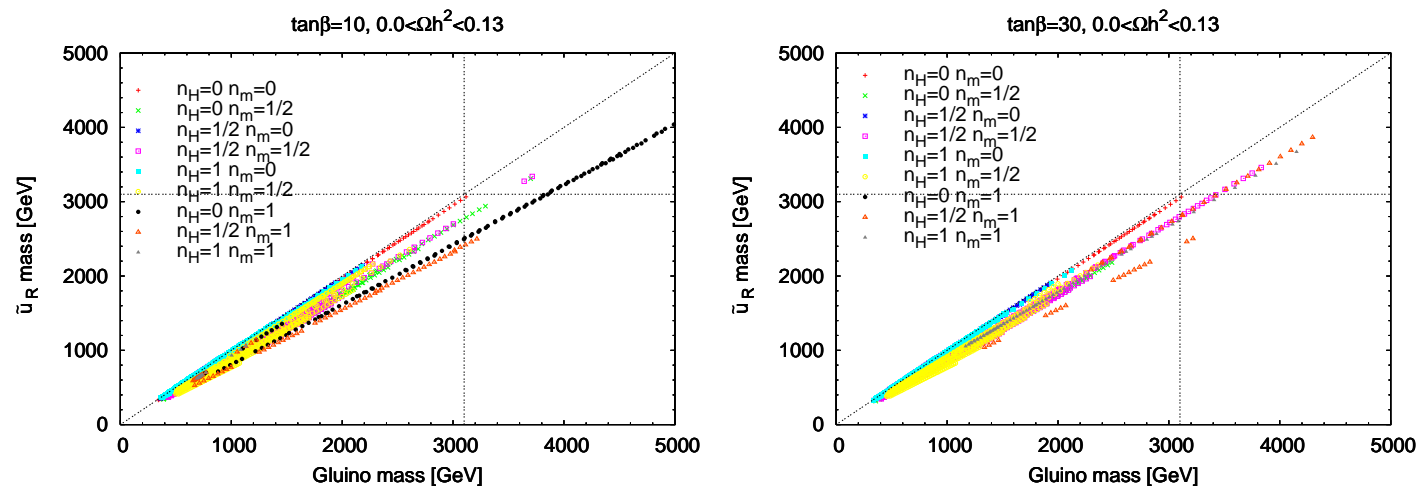

Figure 7: Plot of $m_{\tilde{u}_{R}}$ vs. $m_{\tilde{g}}$ in the MM-AMSB model for various modular weight choices. Only models with $\Omega_{\widetilde{Z}_{1}} h^{2}<0.13$ and LEP2 constraints satisfied are included. In frame $a$ ), we plot models for $\tan \beta=10$, while in frame $b$ ) we plot models for $\tan \beta=30$.
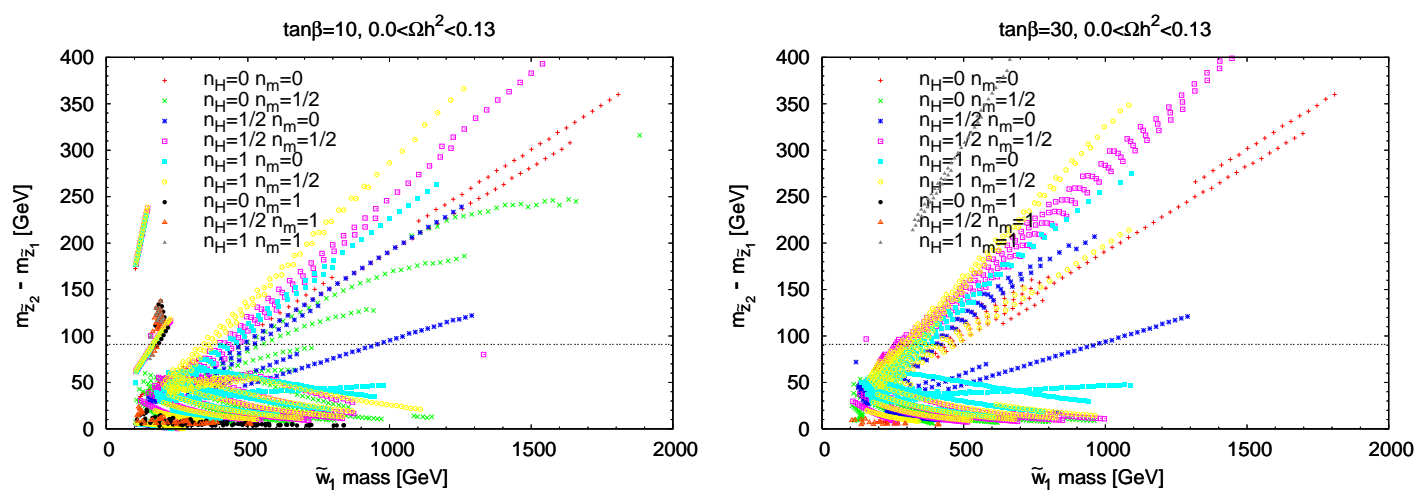

Figure 8: Plot of $m_{\widetilde{Z}_{2}}-m_{\widetilde{Z}_{1}}$ vs. $m_{\widetilde{W}_{1}}$ in the MM-AMSB model for various modular weight choices. Only models with $\Omega_{\widetilde{Z}_{1}} h^{2}<0.13$ and LEP2 constraints satisfied are included. In frame $a$ ), we plot models for $\tan \beta=10$, while in frame $b$ ) we plot models for $\tan \beta=30$.
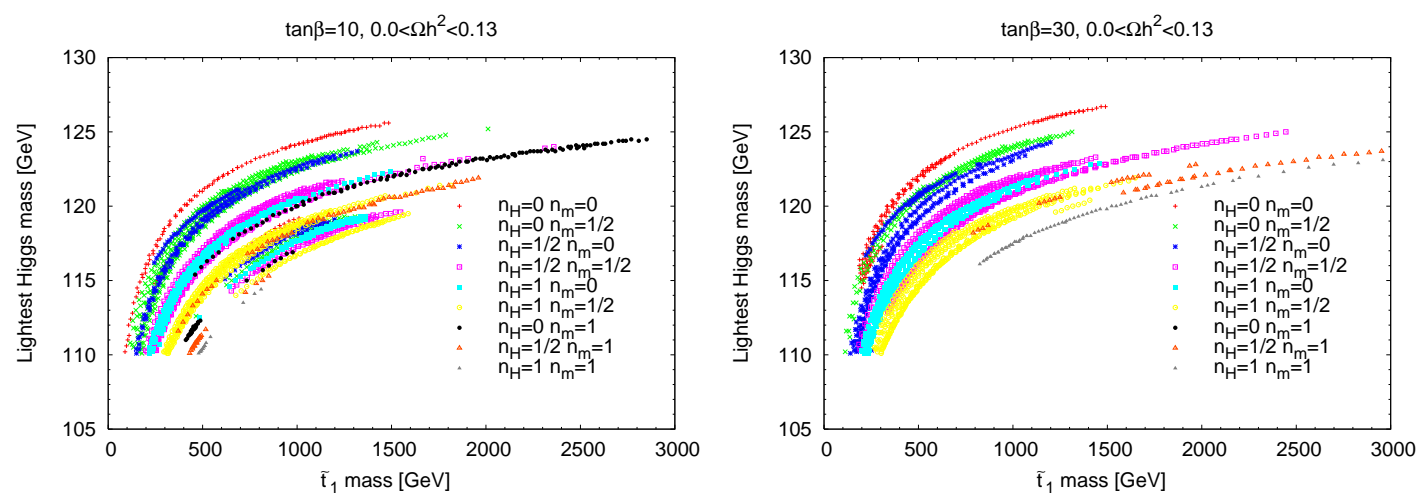

Figure 9: Plot of $m_{h}$ vs. $m_{\tilde{t}_{1}}$ in the MM-AMSB model for various modular weight choices. Only models with $\Omega_{\widetilde{Z}_{1}} h^{2}<0.13$ and consistent with LEP2 constraints satisfied are included. In frame $a$ ), we plot models for $\tan \beta=10$, while in frame $b$ ) we plot models for $\tan \beta=30$. 

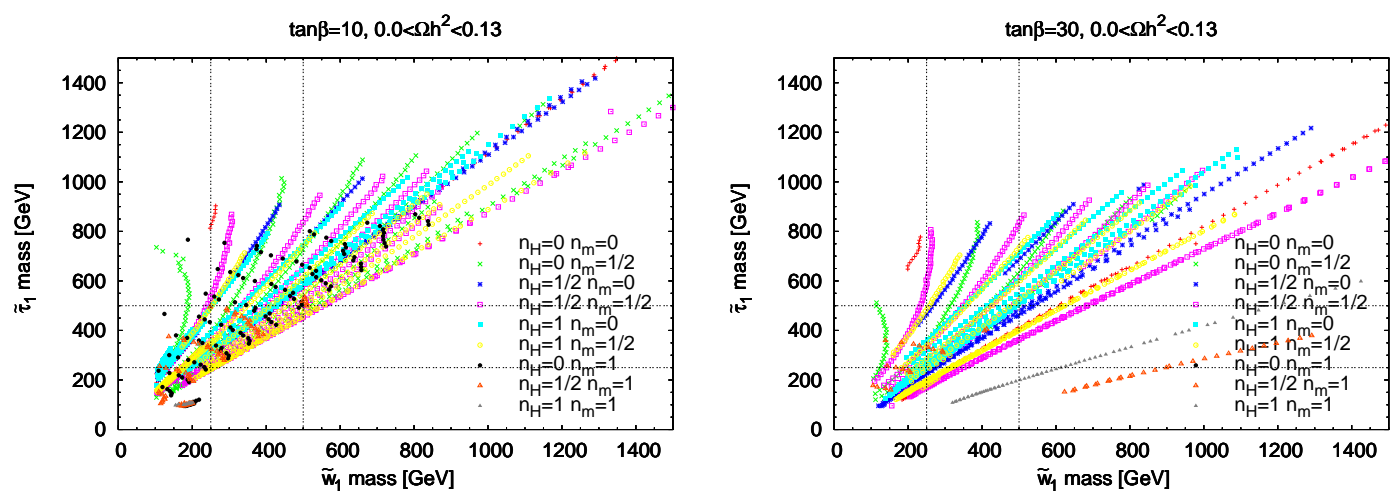

Figure 10: Plot of $m_{\tilde{\tau}_{1}}$ vs. $m_{\widetilde{W}_{1}}$ in the MM-AMSB model for various modular weight choices. Only models with $\Omega_{\widetilde{Z}_{1}} h^{2}<0.13$ and consistent with LEP2 constraints satisfied are plotted. In frame $a$ ), we plot models for $\tan \beta=10$, while in frame $b$ ) we plot models for $\tan \beta=30$.
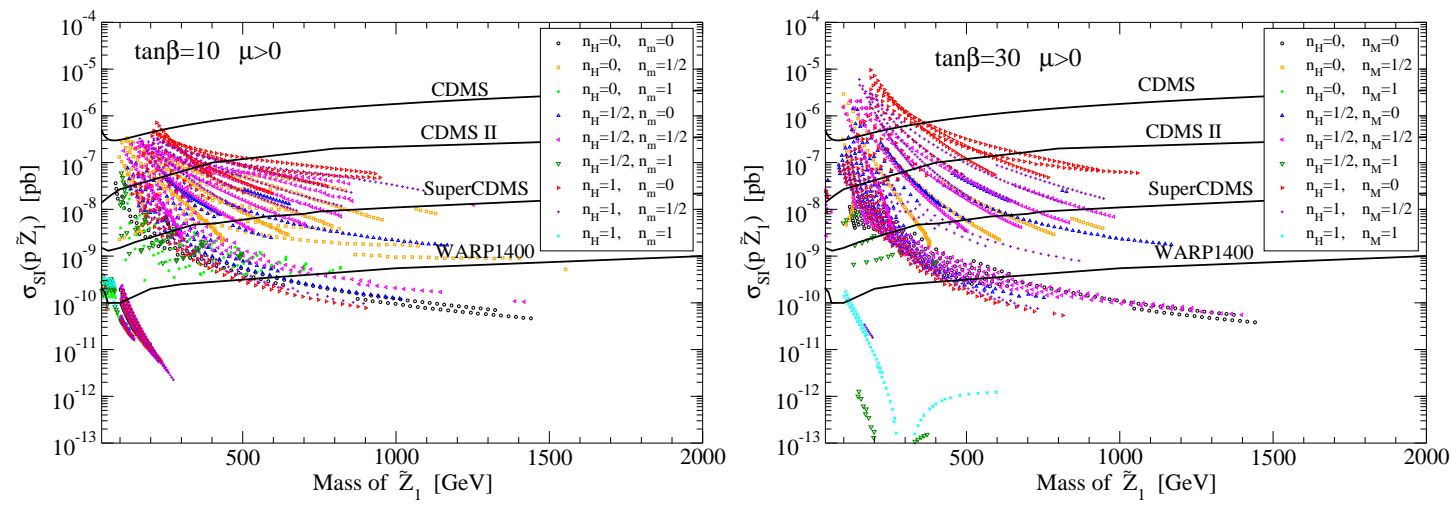

Figure 11: Plot of $\sigma_{S I}\left(\widetilde{Z}_{1} p\right)$ vs. $m_{\widetilde{Z}_{1}}$ in the MM-AMSB model for various modular weight choices for $a) \tan \beta=10$, and $b) \tan \beta=30$. Only models with $\Omega_{\widetilde{Z}_{1}} h^{2}<0.13$ and LEP2 constraints satisfied are included. The region above the contour labelled CDMS is excluded by the non-observation of a signal in the CDMS experiment. The other contours show the projected reach of CDMS (labelled CDMS II) along with the projections for its proposed upgrade (superCDMS) and for the Warm Argon Project (WARP1400), taken here as a representative of proposed ton-sized noble gas detectors. 


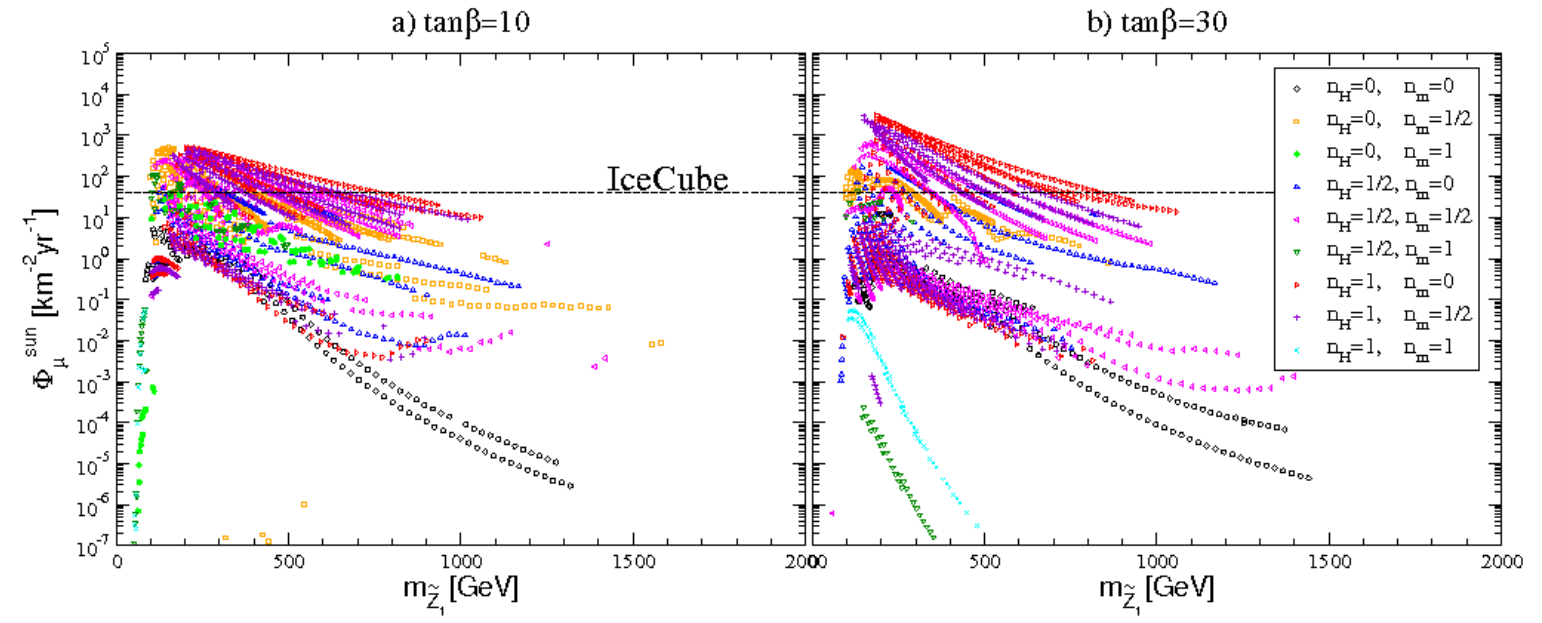

Figure 12: The integrated muon flux and the projection for the ultimate reach of the IceCube experiment for the various choices of Higgs and matter modular weights as labelled on the figure for $a) \tan \beta=10$, and $b) \tan \beta=30$ with $\mu>0$ and $m_{t}=175$. The points above the horizontal dashed line are projected to be within the reach of IceCube, assuming the sensitivity is given by 40 events $/ \mathrm{km}^{2} / \mathrm{yr}$ for $E_{\mu}>50 \mathrm{GeV}$. Only points consistent with constraints from WMAP and LEP2 are included in the figure. 

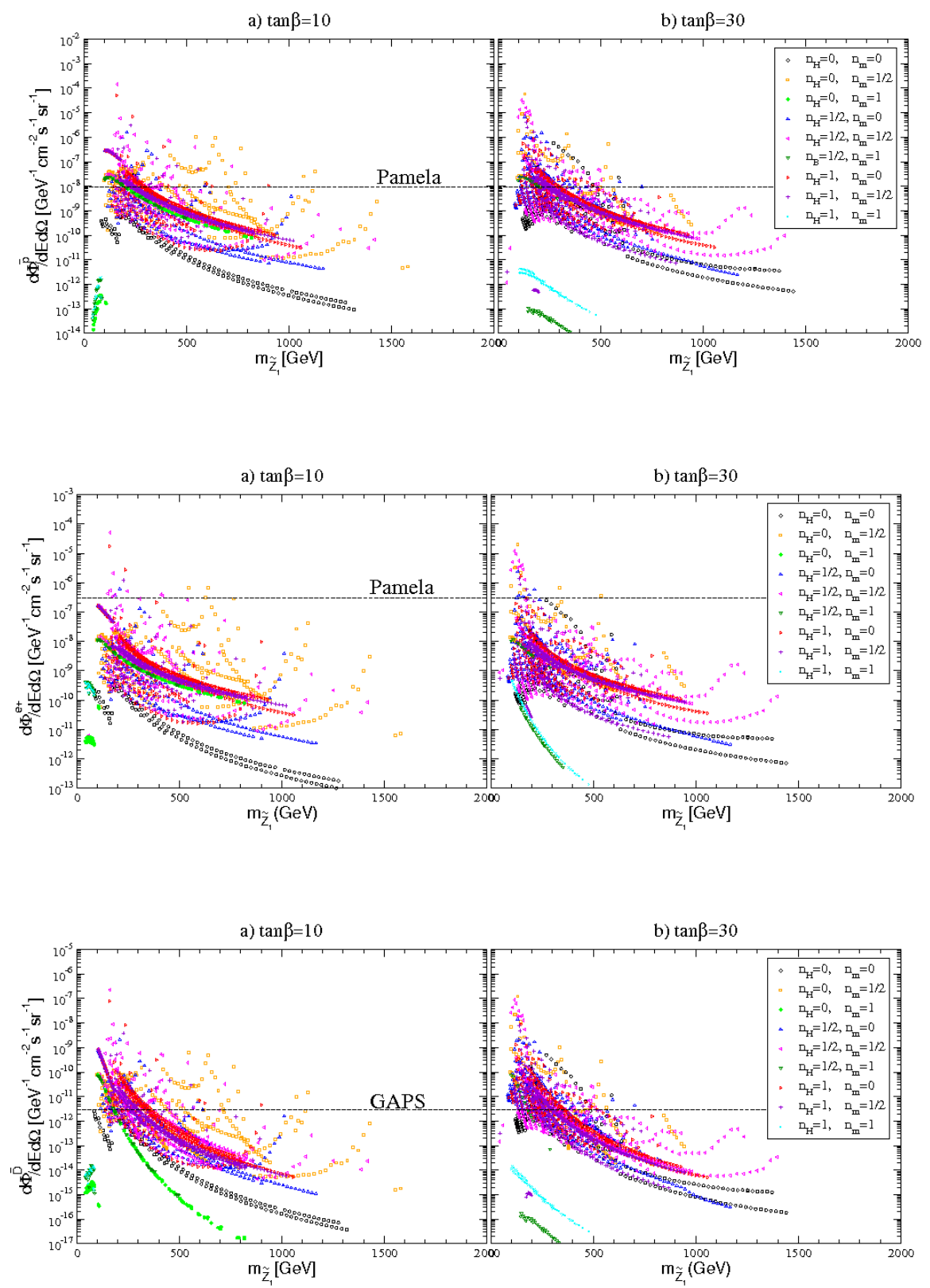

Figure 13: Expected fluxes and projected sensitivities of the Pamela experiment for the detection of anti-protons or positrons, and of the GAPS experiment for the detection of anti-deuterons, from neutralino annihilation in our galactic halo, assuming the Burkert profile for the halo DM distribution for the various choices of Higgs and matter modular weights as labelled on the figure. We take $\mu>0$ and $m_{t}=175$ and show results for $\tan \beta=10$ (left column), and $\tan \beta=30$ (right column). These experiments should be sensitive to points above the horizontal dashed lines with criteria described in the text. Only points consistent with constraints from WMAP and LEP2 are included in the figure. 


\section{Burkert Halo Model}

$\begin{array}{ll}\text { a) Burkert } H M: \tan \beta=10 & \text { b) Burkert } H M: \tan \beta=30\end{array}$

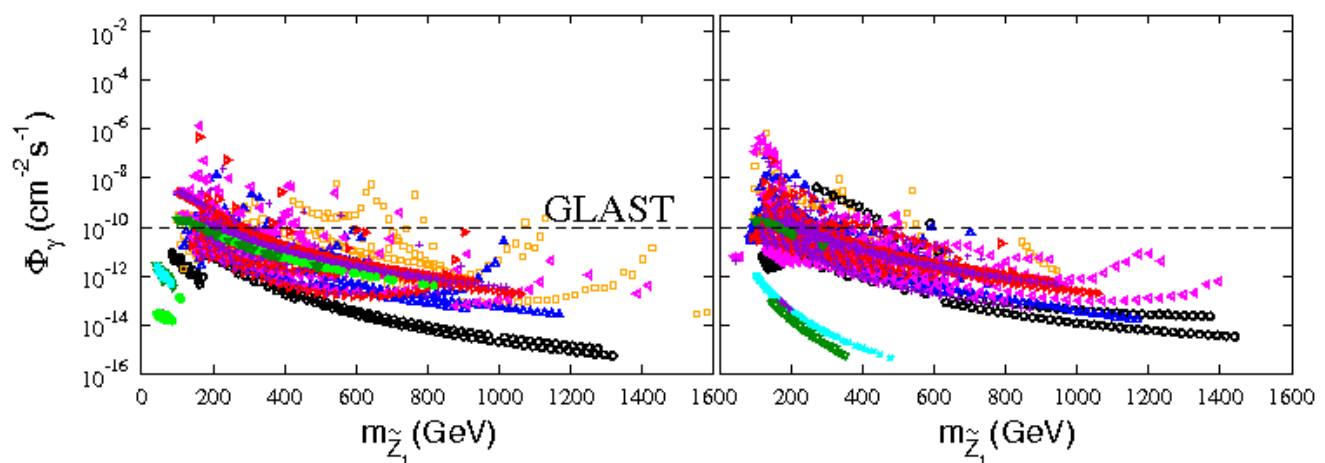

N03 Halo Model

c) $\mathrm{NO} 3 \mathrm{HM}: \tan \beta=10$

d) NO3 HM : $\tan \beta=30$

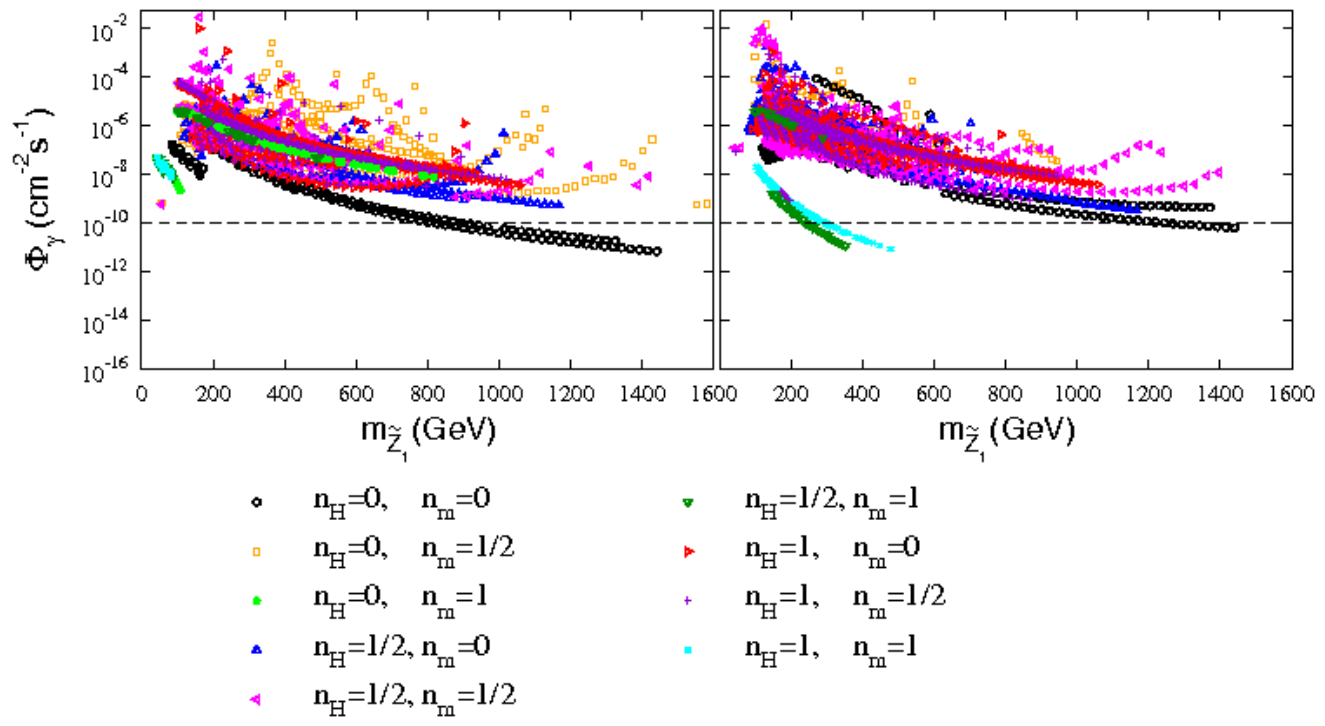

Figure 14: The integrated flux and projected sensitivity of the GLAST experiment for the detection of gamma rays with $E_{\gamma}>1 \mathrm{GeV}$ from neutralino annihilation in the centre of our Galaxy, for the the Burkert profile (upper frames) and for the Adiabatically Contracted N03 halo profile for the galactic DM distribution, for the various choices of Higgs and matter modular weights as labelled on the figure. We take $\mu>0$ and $m_{t}=175$ and show results for $\tan \beta=10$ (left column), and $\tan \beta=30$ (right column). GLAST should be sensitive to points above the horizontal dashed lines with criteria described in the text. Only points consistent with constraints from WMAP and LEP2 are included in the figure. 

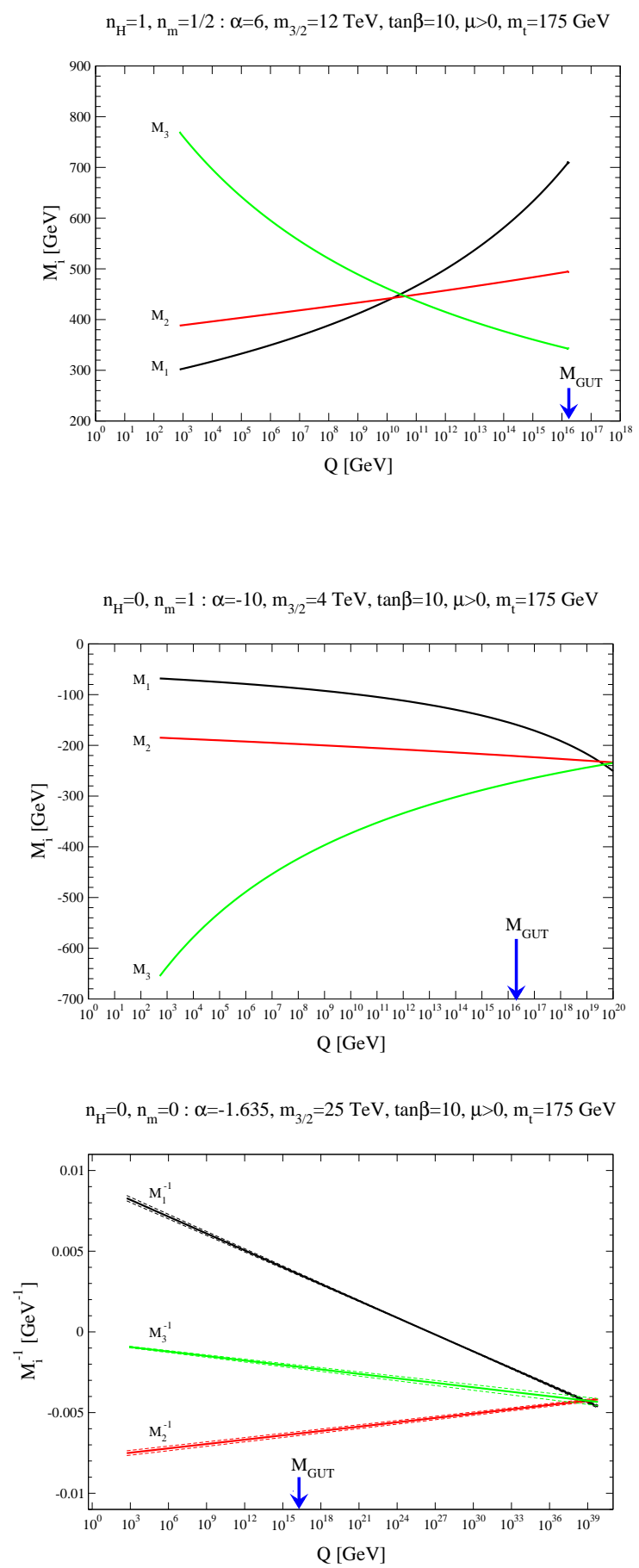

Figure 15: Plot of evolution of gaugino masses versus energy scale $Q$ from $Q=M_{Z}$ to $Q=M_{\mathrm{GUT}}$ and beyond for three cases of MM-AMSB model: $a)\left(n_{H}, n_{m}\right)=\left(1, \frac{1}{2}\right)$ with $\alpha=6$ and $m_{3 / 2}=12$ $\mathrm{TeV}, b)\left(n_{H}, n_{m}\right)=(0,1)$ with $\alpha=-10, m_{3 / 2}=4 \mathrm{TeV}$ and $\left.c\right)\left(n_{H}, n_{m}\right)=(0,0)$ with $\alpha=-1.635$ and $m_{3 / 2}=25 \mathrm{TeV}$. All figures take $\tan \beta=10$ and $\mu>0$. We adopt error projections of $\pm 2 \%$ on $M_{1}$ and $M_{2}$, and $\pm 5 \%$ on $M_{3}$ for frame $c$ ). Note that we continue MSSM evolution even beyond the scale $Q=M_{\mathrm{GUT}}$ for reasons that we discuss in the text. 